\title{
Endcast: mobile stateless data delivery in MANETs
}

\author{
Sulochana Sooriyaarachchi* (D) and Chandana Gamage
}

\begin{abstract}
MANETs are networks of mobile devices for multihop communication without a separate infrastructure for routing and control. If robust protocols are available, MANETs can exploit the true ubiquity of everyday mobile devices for application scenarios such as collaborative learning, multiplayer gaming, adaptive advertising as well as in disaster management and battlefield communication. Traditional schemes such as reactive, proactive and hybrid routing incur costs of often $O\left(n^{2}\right)$ for discovering and maintaining routes or node hierarchies for networks with $n$ number of nodes. These routes or node structures become obsolete rapidly due to MANET dynamics resulting from mobility, limited battery life, and impairments of wireless links. These traditional approaches are based on a paradigm that we refer to as fixed-stateful routing. The routing paradigm that best matches the ubiquity of devices in a MANET is mobile-stateless routing. In mobile-stateless routing, nodes do not keep global network states or play special roles as control nodes. We propose flooding as an end to end packet delivery mechanism and introduce the concept of endcast that allows the communication from a single source to a single destination in which the data is carried by controlled flooding and the data propagation is inhibited after reaching the destination.

Problem statement: Simple flooding causes excessive packet redundancy resulting in collisions and contention which is known as broadcast storm problem. We introduce the term broadcast flood problem to refer to the propagation of data beyond the destination in flooding operation. This research is aimed at modeling endcast and developing an endcast scheme to enable MANET nodes to perform end to end communication in mobile-stateless manner.

Solution: We built a theoretical model to analyze the endcast concept. This model includes methods to quantify broadcast storm problem and broadcast flood problem. Based on this model, we proposed an endcast scheme that controls the storm situation using counter-based flooding and the flood situation using negative acknowledgement packets. The storm control scheme is inspired by the chalone mechanism that regulates growth of biological organs. The proposed scheme is in the mobile-stateless routing paradigm because the nodes keep only a set of local states in the form of a log of received data packets and the nodes do not act as infrastructure nodes. We apply the theoretical model on the proposed endcast scheme to validate its correctness and simulations were done to verify the performance.
\end{abstract}

Keywords: MANET, Multihop, Broadcasts, Flooding

\section{Introduction}

MANETs can be exploited as a ubiquitous computing architecture especially when a dense population of mobile nodes are connected with near field communication. However, the ad hoc mode of operation shows limited or negligible usage compared to infrastructure mode though

*Correspondence: sulochanas@cse.mrt.ac.lk

Department of Computer Science and Engineering, University of Moratuwa, Moratuwa 10400, Sri Lanka it has been in existence in IEEE 802.11 specifications alone for about 20 years. The main cause that hinders true potential of MANETs is the lack of robust multihop communication protocols.

According to Abolhasan et al. [1], asymptotic costs of the proactive, reactive, and hybrid protocols have a memory overhead of magnitude $O\left(N^{2}\right)$ and control overhead of magnitude $O(N)$ ( $N$-number of nodes). However, MANETs are dynamic networks and the routes thus discovered become obsolete rapidly, even before they 
converge in a time of magnitude $O(D)(D$-diameter of network in number of hops). Similarly, the reactive protocol overheads also become higher because the routes become invalid rapidly due to dynamic topology of MANETs. Hybrid protocols require nodes to organize into zones, which also incurs costs apart from the costs of discovering and maintaining routes. Therefore, it is infeasible to route data packets in dense MANETs based on topological states.

Blind rebroadcasting is the simplest mechanism to send a data packet in fully stateless manner such that any node pair in the network can reach each other. However, blind rebroadcasting suffers from severe performance degredation due to massive redundant rebroadcasts resulting in broadcast storm problem. An improved version of blind rebroadcasting is the simple flooding in which a node rebroadcasts a packet only when it is received for the first time. The received packets are checked using a log of packet IDs where each entry comprises of source address and sequence number. There are flooding schemes that evaluate additional variables for making the rebroadcast decision such as counter-based flooding and distance-based flooding. In these flooding schemes, only a subset of nodes that receive the packet will rebroadcast and hence they are known as efficient flooding schemes.

Flooding schemes are conventionally used to disseminate a message to all the members of a network, and this communication mode is known as broadcast. The aim of routing is for a single source to reach a destination using a multihop path, and this communication is referred to as unicast. In this research, we leverage flooding for end to end data delivery where there are no preset point to point paths and only with rebroadcasting at intermediate nodes. We coined the term endcast to reflect this communication mode.

Viswanath and Obraczka [2] analyze flooding in its conventional use of broadcast. Their model includes nodeto-node communication model of $\mathrm{Wu}$ and Varshney [3], multihop propagation of flooding modeled as a graph, and a model for probabilistic flooding. Our work extends these research by modeling efficient flooding schemes such as counter-based flooding. We incorporated mobility and time domain aspects such as random assessment delay (RAD) in our model.

We model endcast using a graph-based representation that we name flooding graph to make insights into the concept. Message propagation in flooding operation is perceived as a flooding wave. We geometrically estimate the area covered by the gradual propagation of a flooding wave. The number of newly reached nodes is estimated by considering node distribution. The characteristics of physical and MAC layer are captured by a probability $\left(P_{S}\right)$ of node-to-node successful transmission using
Markov chain models. The effects of multihop wireless communication such as hidden terminal problem are accounted for in calculating $P_{S}$. The successful reception of a rebroadcast is modeled by another probability $\left(P_{b}\right)$. The properties of efficient flooding schemes are modeled by a probability $\left(P_{t x}\right)$ of rebroadcasting, for example $P_{t x}$ for counter-based flooding is calculated using the counter value and the threshold. Our theoretical model for endcast is capable of (i) modeling the effect of node mobility, (ii) modeling time domain aspects of flooding schemes such as RAD, (iii) modeling efficient flooding schemes, and (iv) quantifying broadcast storm and flood problems.

An endcast protocol should consist of a broadcast storm control mechanism and a broadcast flood control mechanism. We proposed an endcast scheme that controls the storm situation using counter-based flooding and the flood situation using negative acknowledgement packets. The storm control scheme is inspired by the chalone mechanism that regulates the growth of biological organs. An operational model was derived for the scheme using cellular automata with totalistic rules. The protocol was evaluated for reachability using the developed theoretical model, and its performance was analyzed using extensive simulations.

\section{Endcast}

We define endcast as the communication from a single source to a single destination in which the data is carried by controlled flooding and the data propagation is inhibited after reaching the destination. When any kind of flooding is used for end to end data forwarding, waste of network resources occur due to

- Broadcast storm: ideally, only one message should propagate from the source to the destination whereas flooding causes excessive number of messages propagating all over the network. Tseng et al. [4] define this situation as broadcast storm problem.

- Broadcast flood: even after reaching the destination, the messages continue to propagate and get accumulated in the network unless explicit mechanisms are implemented to stop the message propagation and remove the messages. We term this situation as broadcast flood problem.

Traditional approaches of routing in MANETs require global network information or at least neighbor information. Moreover some nodes may act as infrastructure nodes. We consider these approaches as fixed-stateful routing. We name the routing approaches that do not require nodes to maintain global network states or act as control nodes as mobile-stateless routing. Endcast is designed to be in mobile-stateless routing paradigm. 


\section{Related work}

Ho et al. [5] argued that flooding is a possible alternative for reliable multicast in highly mobile ad hoc networks as traditional multicast solutions are not suitable. We propose that flooding is also an alternative for end to end data delivery as it allows to eliminate the cost of discovering and maintaining routes. Rahman et al. [6] also experimentally prove that flooding with tunable heuristics can be adopted as a reliable and efficient routing scheme in MANETs and these flooding-based schemes are not inferior to sophisticated point-to-point forwarding schemes. However, we did not come across any other work that studies utilizing of flooding for end to end communication in MANETs, particularly with a strong theoretical basis.

Viswanath and Obraczka [2] model the propagation of a data packet in a MANET via flooding as a graph rooted at the source. We refer to resulting graph as flooding graph hereafter and use this graph model for theoretical model of endcast. Further, flooding model in [2] consists of node-to-node communication model, multihop communication model, and probabilistic flooding model as explained below.

\subsection{Node-to-node transmission}

Wu and Varshney [3] propose a three-state Markov chain model for node-to-node wireless transmission in the presence of hidden terminal problem and it has been used by Viswanath and Obraczka [2] also. The three states are idle state (I), successful transmission state $(S)$, and collision transmission state $(C)$ as shown in Fig. 1.

Operational details of the MAC layer protocol specify the conditions for a successful node-to-node transmission. Four conditions must be fulfilled for a successful transmission from $x$ to $y$ in Fig. 2 according to CSMA. The notations used for describing these conditions are when $r$ is the distance between nodes $x$ and $y, C(r)$ is the area of the region $N(x) \cap N(y), B(r)$ is the area of the region $N(y)-N(x)$. Takagi and Kleinrock [7] derived Eqs. 1, 2, and 3 for estimating the areas $C(r)$ and $B(r)$.

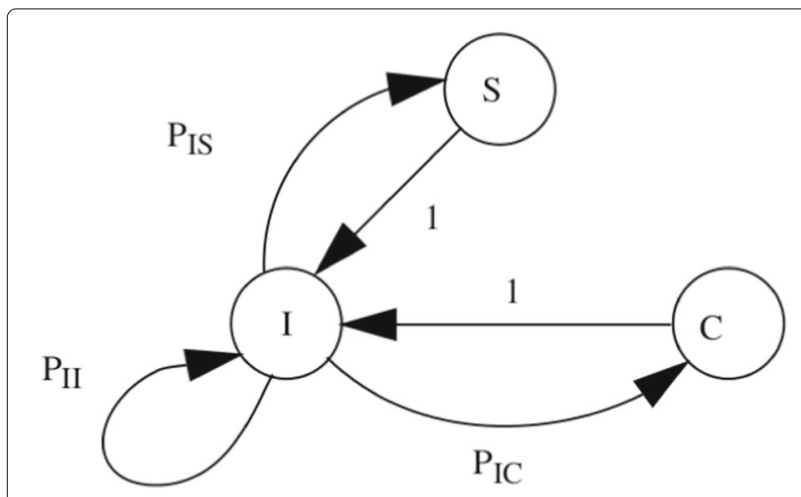

Fig. 1 Three-state Markov chain model of a node [3]

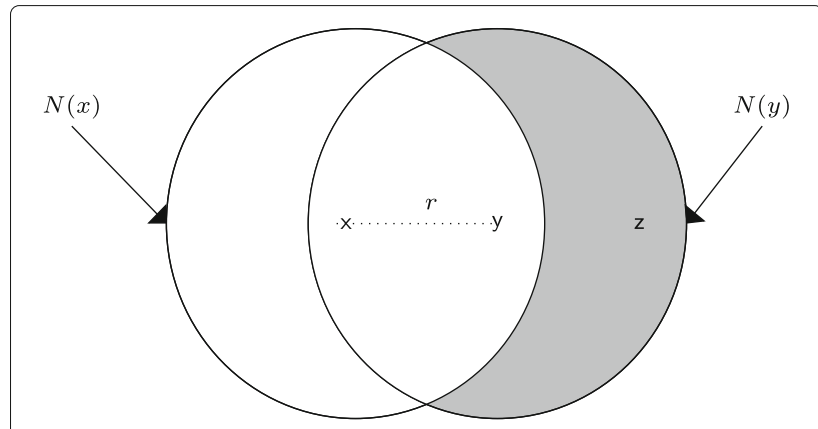

Fig. 2 Illustration of hidden node problem [3]

$$
\begin{aligned}
& C(r)=2 R^{2} q\left(\frac{r}{2 R}\right) \\
& B(r)=\pi R^{2}-2 R^{2} q\left(\frac{r}{2 R}\right) \\
& q(t)=\arccos (t)-t \sqrt{1-t^{2}}
\end{aligned}
$$

Equation 4 gives the conditions for a successful nodeto-node transmission in the presence of hidden terminal problem in CSMA.

$P_{I S}(r)=\left\{\begin{array}{l}\operatorname{Prob}(x \text { transmits in a slot). } \\ \operatorname{Prob}(y \text { does not transmit in the same slor } \mid r) . \\ \operatorname{Prob}(\text { nodes in } C(r) \text { region do not transmit in the same slot } \mid r) . \\ \operatorname{Prob}(\text { nodes in } \mathrm{B}(\mathrm{r}) \text { do not transmit in } 2 \tau+1 \text { slots } \mid r)\end{array}\right.$

\subsection{Multihop flooding}

Multihop flooding model in [2] is explained using Fig. 3. Accordingly, $S$ is the source and $N$ is the average number of nodes in the $S$ 's transmission region. The probability of successful transmission by $S$ to any one of its neighbors is $P_{S}$ from node-to-node transmission model where subscript " $S$ " denotes successful transmission state. The

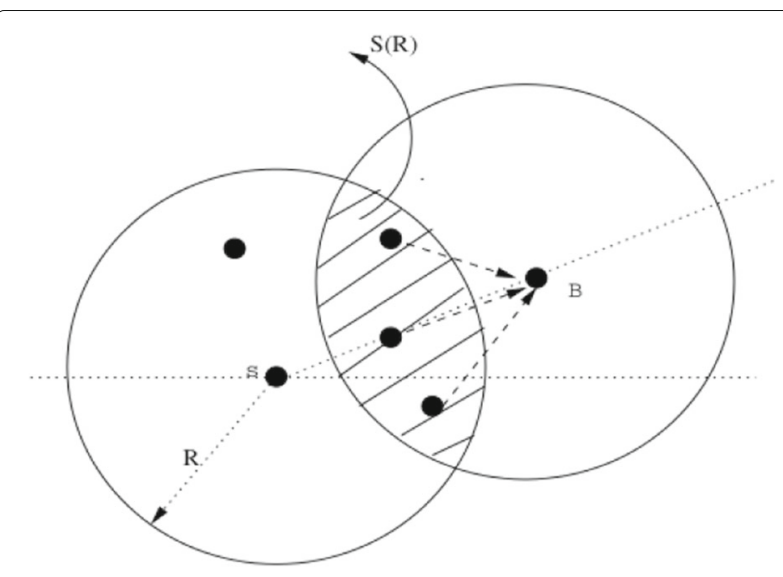

Fig. 3 Second level retransmission [2] 
event of exactly $N_{S}$ number of neighbors successfully receiving the transmission from $S$ is modeled by Binomial probability distribution [2].

$\bar{N}_{S}$ is the number of neighboring nodes that will retransmit the received data. Out of these $\bar{N}_{S}$ nodes, only the retransmissions of nodes in the region $S(R)$ will reach node $B$. Number of nodes in the region $S(R)$ is denoted by $N_{b}$.

$$
\begin{array}{r}
P_{b}=P\left(\text { B receives at least one copy of data } / \bar{N}_{b}\right. \\
\text { nodes transmit })
\end{array}
$$

The probability of successful reception at any retransmission level is $P_{b}$.

It is required to estimate the number of newly reached nodes when a packet is flooded into a network by a single source. The number of nodes $N_{1}$ reached by the original broadcast of the source is given by:

$$
N_{1}=P_{S} N
$$

Number of nodes $N_{2}$ that are newly reached by the rebroadcast of $N_{1}$ nodes is:

$$
N_{2}=\beta P_{S} P_{b} N^{2}
$$

Here, $\beta$ in [2] is the additional coverage due to rebroadcast of receiving nodes at any level. However, we modify $\beta$ as a variable dependent on the level $i$ of rebroadcasting and each additional coverage $\beta_{i}$ is estimated using a geometric method.

\subsection{Modeling probabilistic flooding}

In contrast to simple flooding, a message received for the first time by a node is rebroadcast with a probability $P_{t x}$ in probabilistic flooding. The probability of successful reception at any retransmission level is $P_{b}$ which is derived as follows:

$$
\begin{aligned}
& P_{b}=1-P\left(\text { B receives no copy } / \bar{N}_{b} P_{t x}\right) \\
& P_{b}=1-\left(1-P_{S}\right)^{\bar{N}_{b} P_{t x}}
\end{aligned}
$$

Comparison of our work with existing work is summarized in Table 1.

Table 1 Comparison of our work with Viswanath and Obraczka [2]

\begin{tabular}{lll}
\hline Aspect & {$[2]$} & Our work \\
\hline Probability of successful transmission & From [3] & From [3] \\
Probability of successful reception & Original & From [2] \\
Probabilistic flooding model & Original & From [2] \\
Graph representation of flooding & Original & From [2] \\
Efficient flooding models & $\times$ & Original \\
Flooding wave propagation & $\times$ & Original \\
Effect of mobility on flooding & $\times$ & Original \\
Time domain representation in flooding graph model & $\times$ & Original \\
\hline
\end{tabular}

\section{Theoretical model for endcast}

\subsection{Flooding graph}

In modeling, we first extract the transmission graph for a given node placement. According to Rajaraman [8], in a transmission graph $G=(V, E)$ in Euclidean space, the set $V$ represents all the nodes and set $E$ contains an edge from node $u$ to $v$ if $u$ can directly transmit to $v$. The ability to directly transmit is determined by the path loss equation and signal-to-noise ratio formula [8]. The transmission graph is interpreted as an adjacency matrix. A simple example is given in Fig. 4 for elaborating the graphbased modeling process of endcast. In the flooding graph in Fig. 4c, a broadcast of node $A$ is received by the nodes that are adjacent to $A$ (the neighbors of $A$ ). The number of frames accumulated in the network is equal to the number of edges in the graph. Number of edges in turn depends on MANET parameters such as network topology, neighborhood and wireless transmission characteristics.

A generalized flooding graph is given in Fig. 5 where $N_{x y}$ denotes the nodes that are $x$ hops away from the source and $y$ is the index for nodes at that level. Further, $x$ represents the rebroadcast level, for example the immediate neighbors (one hop neighbors) of the source will rebroadcast the packet for the first time and thus become level 1 rebroadcasters. Each edge $(u, v)$ represents the reception of a packet by a node $v$ due to a broadcast of a node $u$.

Equation 7 gives the number of nodes reached by each level of rebroadcast. We modify Eq. 7 by replacing $\beta$ with $\beta_{i}$ resulting in follows expressions.

$N_{1}$ is the number of nodes reached by the transmission of the source, given by:

$$
N_{1}=P_{S} N
$$

$N_{2}$ is the number of nodes newly reached by the second transmission, given by:

$$
N_{2}=\beta_{1} P_{t x} P_{b} N N_{1}
$$

$N_{l}$ is the number of nodes newly reached by the $l^{\text {th }}$ retransmission, given by:

$$
N_{l}=\beta_{l-1}\left(P_{t x} P_{b} N\right)^{l-1} P_{S} N
$$

Therefore, $N_{T}$ is the reachability, given by:

$$
N_{T}=P_{S} N+P_{S} N \sum_{i=1}^{l-1} \beta_{i}\left(P_{t x} P_{b} N\right)^{i}
$$

\subsection{Geometric analysis of message propagation in flooding}

We perceive the propagation of the data frame via flooding as a flooding wave as shown in Fig. 6a. When node A initiates a flooding operation, A's neighbors rebroadcast such that the nodes in the dotted area receive the frame. These newly reached nodes will rebroadcast at the next level to reach another set of new nodes. This process can 


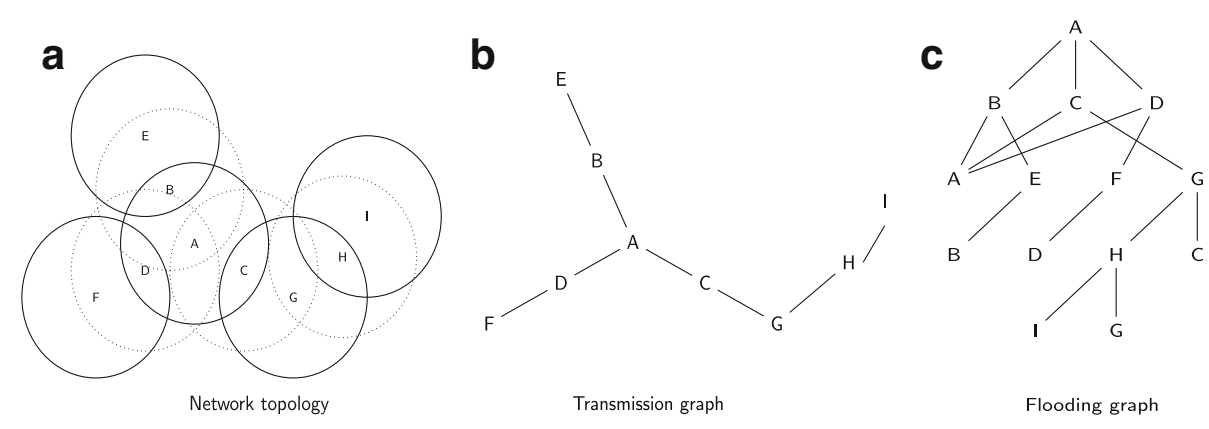

Fig. 4 Extracting the flooding graph of a MANET. a Network topology. b Transmission graph. c Flooding graph

be visualized as similar to how a drop of water fallen on a static pool of water causes a water ripple that propagates outwards as in Fig. 6b.

Consider a scenario in which a node $A$ broadcasts a message and another node $B$ that receives it decides to rebroadcast. Figure 7 represents this scenario and the shaded area will be the additional area that can benefit from the transmission of B. If $S_{A}$ and $S_{B}$ are the circular areas covered by A and B respectively, then $S_{B-A}$ (the shaded area in Fig. 7) is given by $S_{B-A}=S_{B}-S_{A \cap B}=$ $\pi r^{2}-\operatorname{INTC}(d)$, where $\operatorname{INTC}(d)$ given by Eq. 14 is the intersection of $S_{A}$ and $S_{B}$ as derived in [4].

$$
\operatorname{INTC}(d)=4 \int_{\frac{d}{2}}^{r} \sqrt{r^{2}-x^{2}} d x
$$

If $B$ is randomly located in the transmission region of $A$, the expected additional coverage (EAC) is given by Eq. 15 where $r$ is the transmission range of each node. By solving Eq. 15, EAC is obtained as $41 \%$ [4].

$$
E A C=\int_{0}^{r} \frac{2 \pi x\left[\pi r^{2}-\operatorname{INTC}(x)\right]}{\pi r^{2}} d x
$$

\subsubsection{Nodes covered by the first level of rebroadcast}

We have to estimate average increment of area $b$ by rebroadcast of all the neighbors of A (dotted area in Fig. 6a). The average additional area covered by the rebroadcast of a single neighbor is $41 \%$ of radio range [4] (dark gray area in Fig. 8). The total effective additional coverage areas by the rebroadcast of all neighbors of A equal to $b$. We estimate $b$ by considering the number of radio range circles needed to cover the circumference of the radio range circle of A. An arc of length $l$ will be covered by a single neighbor. Then the number of arcs required to cover radio range circle equals $\frac{2 \pi R}{l}$. Here, $l=R \theta$.

From Fig. 8,

$$
\begin{aligned}
& \theta=2 \cos ^{-1} \frac{d}{2 R} \\
& l=2 R \cos ^{-1} \frac{d}{2 R}
\end{aligned}
$$

Therefore, the number of arc lengths $k$ that will cover the circumference of a circle of radius $R$ is given by $2 \pi R / l$ which will be simplified to Eq. 18;

$$
k=\frac{\pi}{\cos ^{-1} \frac{d}{2 R}}
$$

Since the average additional area covered by rebroadcast of a single neighbor is $0.41 \pi R^{2}$ [4] the average additional area $b$ covered by all neighbors of $\mathrm{A}$ will be given by $0.41 k \pi R^{2}$ only if the maximum number of neighbors (highest node degree) $d e g_{\max } \geq k$, which simplifies to Eq. 19;

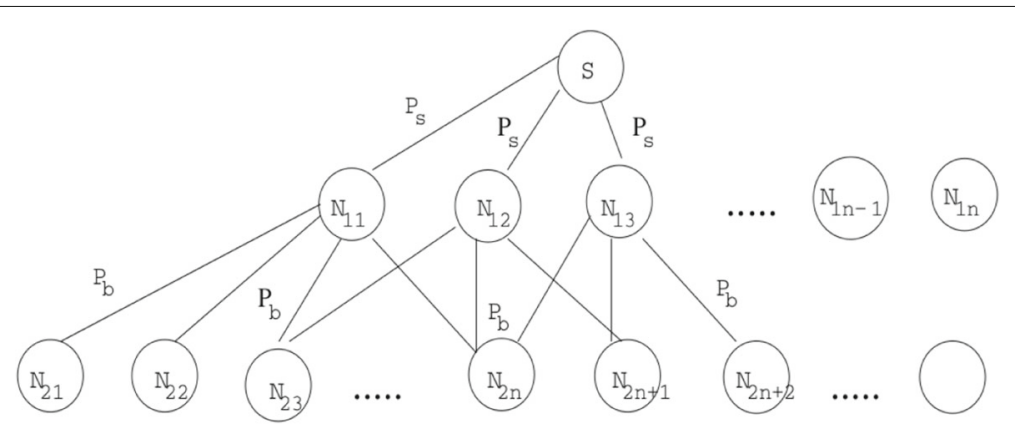

Fig. 5 First two retransmissions of flooding [2] 


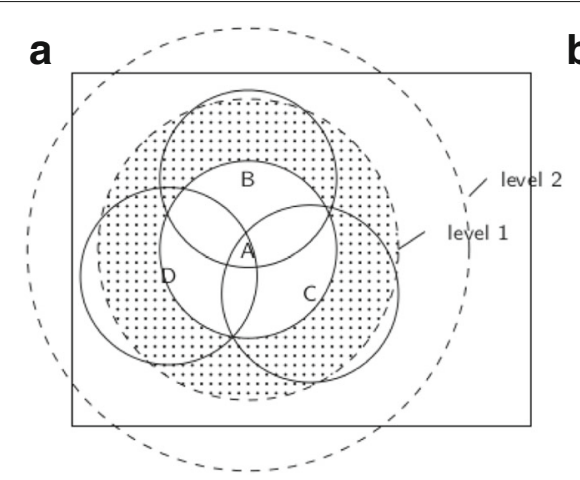

b

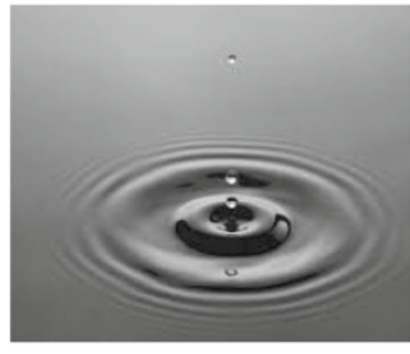

Fig. 6 Geometric estimation of reached nodes at each rebroadcast level. a Illustration of flooding wave. b Water ripple

$$
b=\left\{\begin{array}{l}
0.41 k \pi R^{2}, \operatorname{deg}_{\max } \geq k \\
0.41 d e g_{\max } \pi R^{2}, d e g_{\max }<k
\end{array}\right.
$$

The term $d$ in Eq. 18 denotes the distance between $\mathrm{A}$ and any neighbor $\mathrm{B}$ and can vary from 0 to $\mathrm{R}$. The lower bound of $b$ is when $d=0$ whereas the upper bound is when $d=R$. Average distance $d_{a v}$ corresponds to the case that the transmission ranges of A and B overlap by $59 \%$ of area as EAC is computed to be $41 \%$.

According to Fig. 9b total area of the wedge is $\frac{R^{2} \theta}{2}$ and $A_{1}=\frac{R^{2} \theta}{2}-A_{2}$.

$$
\begin{aligned}
& A_{2}=\frac{R d \sin \frac{\theta}{2}}{2} \\
& A_{1}=\frac{R^{2} \theta-R d \sin \frac{\theta}{2}}{2}
\end{aligned}
$$

Intersection of transmission ranges of $\mathrm{A}$ and $\mathrm{B}$ denoted by $b^{\prime}=2 A_{1}$.

$$
b^{\prime}=R^{2} \theta-R d \sin \frac{\theta}{2}
$$

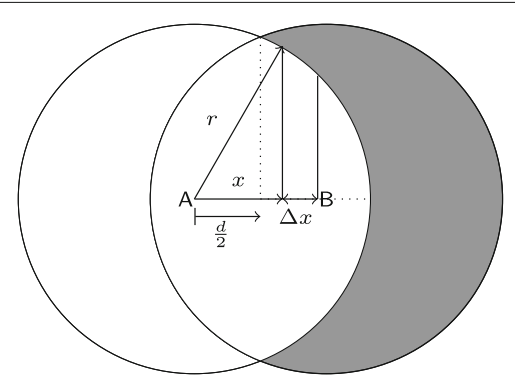

Fig. 7 Additional area covered by a rebroadcast [4]

$$
\cos \frac{\theta}{2}=\frac{d}{2 R}
$$

$$
\sin \frac{\theta}{2}=\sqrt{1-\frac{d^{2}}{4 R^{2}}}
$$

Using $\cos \theta=2 \cos ^{2} \frac{\theta}{2}-1$;

$$
\cos \theta=\frac{d^{2}}{2 R^{2}}-1
$$

Substituting in Eq. 22;

$$
b=2 R^{2} \cos ^{-1}\left(\frac{d}{2 R}\right)-\frac{d}{2} \sqrt{4 R^{2}-d^{2}}
$$

Equation 22 should be solved for $b^{\prime}=0.59 \pi R^{2}$ to calculate average distance, $d_{a v}$;

$$
0.59 \pi R^{2}=2 R^{2} \cos ^{-1}\left(\frac{d_{a v}}{2 R}\right)-\frac{d_{a v}}{2} \sqrt{4 R^{2}-d_{a v}^{2}}
$$

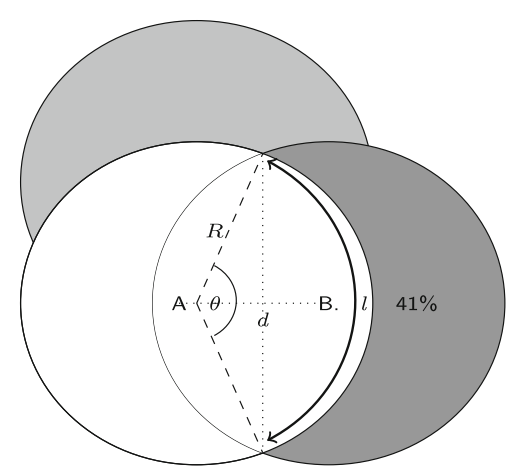

Fig. 8 Estimating the average area covered by rebroadcast level 1 


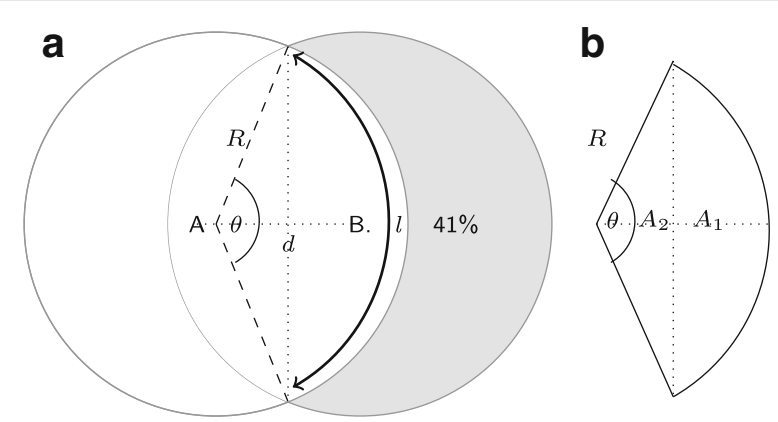

Fig. 9 Estimating average distance $d_{a v}$ between source and a neighbor. a Intersection between transmission ranges. b Calculating area of wedge

The average number of nodes newly reached at rebroadcast level 1 by the flooding wave denoted as the discrete random variable $n$ was calculated using Eq. 28 and $p(n)$ is the probability that there exists $n$ number of nodes in a considered region. The probability $p(n)$ is calculated assuming Poisson node distribution given by Eq. 29 .

$$
\begin{aligned}
& E[n]=\sum_{n=0}^{\infty} n p(n) \\
& P\{N(a+b)-N(a)=n\}=e^{-\lambda b} \frac{(\lambda b)^{n}}{n !}, n=0,1, \ldots
\end{aligned}
$$

\subsubsection{Nodes covered by next levels of rebroadcast}

The additional area covered by rebroadcast level 1 is added to the coverage of the source to get the area covered by level 1 circle in Fig. 6a. This gives Eq. 30 where $b$ is given by Eq. 19 .

$$
\begin{aligned}
& A_{\text {level1 }}=\pi R^{2}+b \\
& R_{\text {level1 }}=\sqrt{\frac{A_{\text {level1 }}}{\pi}} \\
& C_{\text {level1 }}=2 \pi R_{\text {level1 }} \\
& k_{\text {level2 }}=\frac{\pi R_{\text {level1 }}}{R \cos ^{-1} \frac{d}{2 R}}
\end{aligned}
$$

The circumference $C_{\text {level1 }}$ of level 1 circle is calculated from the area of the circle using Eqs. 30, 31, and 32. Then, the number of arc lengths of radius $\mathrm{R}$ that covers the circumference referred to here as $k_{\text {level2 } 2}$ is estimated using Eq. 33. The additional area $b_{2}$ that the second rebroadcast will cover is estimated using Eq. 19.

The probabilities that there are 1 through $n_{\text {total }}$ number of nodes residing in the area $b_{2}$ are calculated using Eq. 29 to obtain the probability mass function (PMF). Thereafter, the number of newly reached nodes by the second rebroadcast is obtained using Eq. 28. This process is repeated for all the other levels of rebroadcasts until all the nodes have finished rebroadcasting or discarding the message for the first time.

\subsection{Model validation}

To validate the model, an analysis was done on a sample network consisting of 20 nodes that were randomly placed on a $85 m \times 85 m$ playground with each node having a transmission range of $40 \mathrm{~m}$. The analytical results shown in Table 2 were obtained after applying the graphbased model with geometric analysis on this network in ideal wireless conditions of no collisions. The number of messages accumulated in the network was also calculated by building the flooding graph shown in Fig. 4c. The graph was built using actual neighborhood sizes that were obtained from the simulation.

The total number of packets accumulated in the network by the time each rebroadcast level finishes is shown in Fig. 10. As evident from the graph, this number grows exponentially. Hence, both the theoretical results and the simulation results show the same behavior. Theoretically estimated number of packets grows at a lower rate than shown in simulation results. This outcome is due to our use of average node degree $\overline{d e g}$ in place of actual node degrees in the theoretical model.

\subsection{Modeling efficient flooding schemes}

In efficient flooding schemes, only a subset of receiv-

\begin{tabular}{|c|c|c|c|c|c|}
\hline Level i & Area covered $A_{i}\left(m^{2}\right)$ & Rebroadcast radius $R_{i}(m)$ & $\begin{array}{l}\text { No. of arc lengths on cir- } \\
\text { cumference } k_{i}\end{array}$ & $\begin{array}{l}\text { No. of new nodes } \\
n_{i}\end{array}$ & $\begin{array}{l}\text { No. of total edges } \\
E\end{array}$ \\
\hline 0 & 5027 & 40 & 2.53 & $3\left(=d_{s r c}\right)$ & 3 \\
\hline 1 & 10,249 & 57 & 3.62 & 6 & 22.2 \\
\hline 2 & 17,706 & 75 & 4.77 & 6 & 61.5 \\
\hline 3 & 27,507 & 94 & 5.93 & 4 & 100.8 \\
\hline
\end{tabular}
ing nodes will rebroadcast a packet after evaluating a set of conditions. For example, in counter-based flooding, a node counts the number of times the same packet is received and checks it against a threshold. The relative

Table 2 Theoretical results for flooding propagation for 20-node sample network

Transmission radius $=40 \mathrm{~m}$; average distance from a transmitting node to a neighbor $=26 \mathrm{~m} ; \theta$ when average additional coverage is $41 \%=2.48$ radians $\left(142^{\circ}\right)$; $\overline{\operatorname{deg}}$ average node degree $=6.4$ 


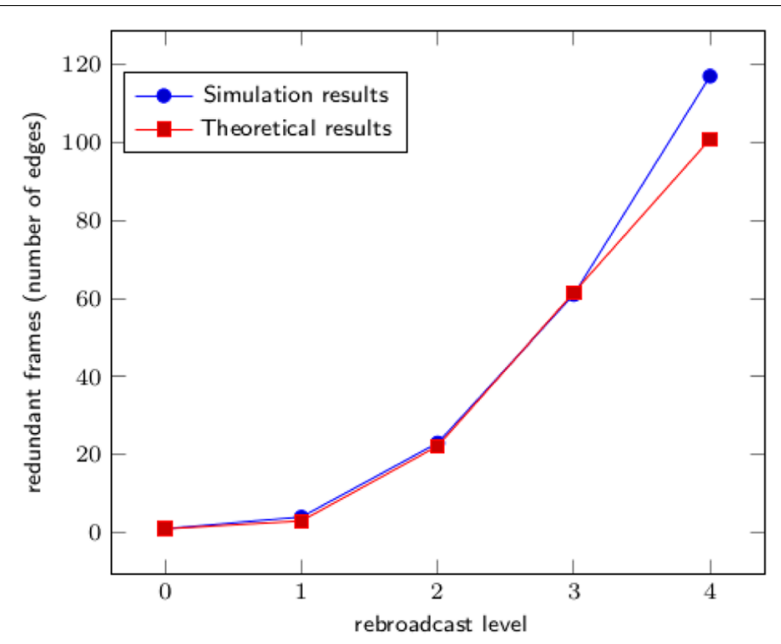

Fig. 10 Redundant rebroadcast frames with rebroadcast levels in simple flooding for a sample 20-node network

distance from the sender nodes against a threshold value is used in distance-based schemes. The assessment of variables representing conditionals for rebroadcast decision requires a time interval for the nodes to gather required neighbor information. This time interval, termed Random Assessment Delay (RAD) by Williams and Camp [9] is denoted as $T_{R A D}$.

The nodes at each level in the simple flooding tree are actual rebroadcasters. In efficient flooding schemes, these nodes are tentative rebroadcasters and would rebroadcast depending on their physical location relative to the source.

In contrast to the case of simple flooding, the graph model for efficient flooding requires the incorporation of time dimension. We index the set of edges with the time a packet is received by the receiving node so that we can determine which set of neighbors will rebroadcast upon the expiry of $T_{R A D}$. The set of nodes that actually rebroadcast is decided by the assessed variable against given criteria within $T_{R A D}$ of that node. The order in which $T_{R A D}$ expires for nodes in the same level determines the different configurations of the tree.

We represent parameters governing rebroadcast decisions in efficient flooding schemes in terms of $P_{t x}$. For example, according to the specification of counter-based flooding it is required that $C<K$ for a node to rebroadcast where $C$ denotes the counter and $K$ denotes the threshold. Therefore, $P_{t x}=P\{C<K\}$ for counter-based flooding. In order to estimate $P_{t x}$, the assessed variable such as counter in counter-based flooding is interpreted as a probability mass function.

\subsection{Modeling node mobility}

An impact of mobility is that the wireless links exist as long as the transmitter and the receiver do not move away from the communication ranges of each other. The probability of completing a transmission of a data packet between two moving nodes is derived by Cho and Hayes [10]. This probability should be incorporated in calculating probability $P_{S}$ of successful node to node transmission for analyzing impact of mobility at a micro level.

However, as we model mobility at macro level, it is done by considering the impact of mobility on the variation of the variable assessed for making the rebroadcast decision. For example, the probability mass function of counter in counter-based flooding is analyzed for impact of mobility and $P_{t x}$ is calculated as a mobility-dependent parameter.

\section{Proposed endcast scheme}

We started with blind rebroadcasting, which is the simplest mobile-stateless routing mechanism and cell proliferation was identified as an analogy to blind rebroadcasting. A grown organ in human body is made out of a dense population of biological cells. The organs originate from a small number of stem cells and these cells divide themselves repetitively in a process called cell proliferation [11-13]. The mapping we have created between cell proliferation and re-broadcasting of packets in a MANET is given in Table 3.

It is an interesting question to ask, "On what basis does our ears end up growing into a particular size?". The nature has its regulatory mechanisms for controlling the cell division in an organ whereby individual cells sense the growth in terms of number of accumulated cells. This is achieved using the concentration of some secreted molecules known as chalones [11]. Bullough [14] coined the term "chalone" and hypothesized it as the inhibitors of cell growth by negative feedback to control the size of a tissue [15-18].

Table 3 Comparison of cell proliferation with blind rebroadcasting

Cell proliferation Blind rebroadcasting

It is local to the micro environment Each node has no state information of the cells and uses information about the topology or the either by direct signaling or by neighborhood of the nodes indirect signaling via the micro environment

Cells are identical to each other and Every node performs the same goes through the same life cycle simple task of forwarding a received data packet

Each cell divides into two so that Total number of packets in the the number of cells should grow network grows exponentially exponentially if there are no

regulatory mechanisms

Cells are organized into a dense Large number of interacting nodes population in a biological organ in the network 
Our endcast scheme is inspired by this chalone mechanism and is targeted to operate at link layer with a frame as the unit of transmission.

\subsection{Biological system to MANET mapping}

We map a biological cell to a data frame in a node as shown in Table 4. While cells increase in number due to proliferation, the frames in a MANET increase in number due to rebroadcasts. However, while the number of cells in the organ is represented by the chalone concentration, the total number of accumulated frames cannot be known using a similar local mechanism, although increase in the frame count depends on the neighborhood of the rebroadcasting nodes. Therefore, the growth of the number of frames is indirectly estimated using the number of neighbors.

A conventional method to obtain neighborhood information in MANETs is to periodically exchange beacon frames. However, beaconing is undesirable in dense MANETs due to increased collisions and contention. Therefore, the received frame count acts as the chalone concentration to indicate the total accumulated frame count in the network. For instance, if a node receives the same frame from many neighbors, that node may not reach new nodes by rebroadcasting the same frame.

A node decides to rebroadcast a received frame depending on the number of redundant copies of the same frame received from elsewhere. If the count of redundant frames reaches a threshold the node decides not to rebroadcast. This is similar to chalone mechanism and is already known as the counter-based flooding mechanism in the context of communication networks. We borrow the term chalone for the actions regarding the neighborhood in MANETs to denote the inspiration from chalone mechanism in cell proliferation.

\subsection{Operational model of the bio-inspired system}

In general, the functional behavior of cell biological systems are modeled in terms of cellular automata (CA) as in [19-22]. Similarly, the operation of biologically inspired rebroadcasting model for MANETs is interpreted based on cellular automata in this section.

Table 4 Mapping from biological system to MANET

\begin{tabular}{ll}
\hline Biological system & MANET \\
\hline Biological cell & Data frame in a node \\
Cell division & Frame rebroadcasting \\
Chalone & The received data frames from the \\
& neighbors. \\
& Neighbors are the nodes within the \\
& transmission range of a node
\end{tabular}

Chalone concentration Number of neighboring nodes who have already broadcast the data frame
According to [23], a two-dimensional CA consists of a regular lattice of sites. Each site takes on $\mathrm{k}$ possible values. Each site is updated in discrete time steps according to a set of rules $\phi$ that depends on the values of sites in some neighborhood around it at the previous time step. Packard and Wolfram [23] address the special class of $\phi$ known as totalistic rules in which the values of a site depends only on the sum of values of the neighbors.

Our CA model of MANET is also viewed as a regular lattice on which mobile nodes are randomly placed. Each site takes only two values: 1 to denote that the node has already rebroadcast a specified data frame and 0 to represent that the node has not rebroadcast. Neighborhood for our CA is the circular region captured by the radio range of a mobile node. Any node within the range will respond as a neighbor. We apply totalistic rule in which we count the total number of neighbors who have rebroadcast a specific data frame. The number of neighbors who have rebroadcast (state 1 ) is given by the number of frames received from the neighborhood. If this number exceeds a predefined threshold a node can decide not to rebroadcast a data frame in hand and we name this predefined value as chalone threshold. This will be the cellular automata rule.

We designed the simulation model for the protocol in a modular architecture by considering the cellular automata-based representation of the protocol. Each node independently runs the same simple set of actions (Fig. 11): if a frame is received for the first time then buffer it; wait for a $T_{R A D}$; count the number of frames received subsequently; when $T_{R A D}$ elapses evaluate the rule "counter is less than the threshold?"; if less then rebroadcast (state 1) else discard (state 0).

\subsection{Protocol architecture and design}

The protocol consists of two phases: storm control mechanism and the flood control mechanism.

\subsubsection{Storm control mechanism}

Our conservative frame rebroadcast scheme termed the storm control mechanism operates as follows: when a node receives a data frame for the first time, it enqueues the frame and creates a record in its frame log. Then, it waits for a time period termed chalone wait listening for the neighbor information. If the same data frame is received subsequently during the chalone wait then a counter incremented for that frame. Thus, the log record includes the number of neighbors who have already rebroadcast the frame. When the chalone wait time elapses, the node evaluates the number of rebroadcast neighbors to see whether it exceeds a pre-defined threshold that we name chalone threshold. If the number of rebroadcasting neighbors does not exceed the threshold, then the node dequeues the frame by rebroadcasting it. Otherwise, the 


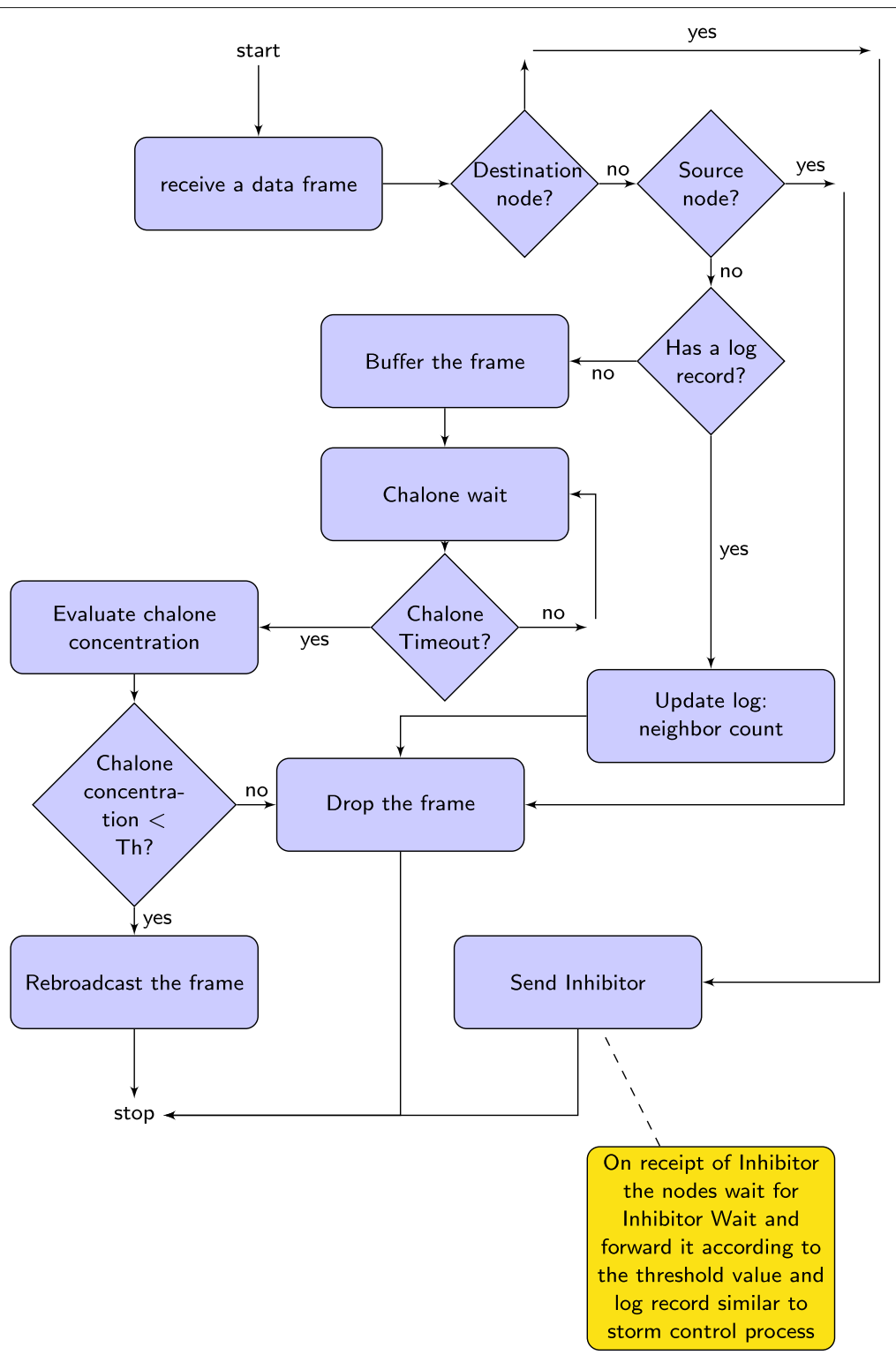

Fig. 11 Event flow at a receipt of a data frame by a node

frame gets discarded. According to the specification of counter-based scheme in [4], our approach can also be characterized broadly as counter-based flooding.

The forwarding decision is made per frame basis by referring to the frame log of the form shown in Fig. 12. A data frame is uniquely identified by the combination of source MAC, destination MAC, and a sequence number generated by the source which altogether is considered as the Frame ID. Data state carries the information such as whether the data is discarded or rebroadcast. Control state is to record any control message sent or received about the frame.

\subsubsection{Flood control mechanism}

Although the above threshold-based mechanism rebroadcasts the data frame in a conservative manner, the frame

\begin{tabular}{|l|l|l|l|l|l|l|l|}
\hline $\begin{array}{l}\text { time } \\
\text { stamp }\end{array}$ & $\begin{array}{l}\text { source } \\
\text { MAC }\end{array}$ & $\begin{array}{l}\text { destination } \\
\text { MAC }\end{array}$ & $\begin{array}{l}\text { seq num- } \\
\text { ber }\end{array}$ & $\begin{array}{l}\text { data } \\
\text { state }\end{array}$ & $\begin{array}{l}\text { inhibitor } \\
\text { state }\end{array}$ & $\begin{array}{l}\text { inhibitor } \\
\text { count }\end{array}$ & $\begin{array}{l}\text { neighbor } \\
\text { count }\end{array}$ \\
\hline
\end{tabular}

Fig. 12 Log format 
will continue to flood outwards in the MANET starting from the source node. Therefore, when it is received by the intended destination, the flooding wave must be stopped.

Our adaptive frame inhibition scheme which we call the flood control mechanism operates as follows: a node sends a control frame named inhibitor as soon as it receives a data frame destined to itself. Any other node that receives an inhibitor forwards it only if there exists a record of the data frame that the inhibitor corresponds to. In order to reduce the number of inhibitors forwarded by nodes, the storm control mechanism above is followed. However, the wait time referred in this case as inhibitor wait is considerably low compared to chalone wait time. Thus, the proposed inhibition scheme is initiated by the destination and the inhibitors propagate faster than the flooding wave only for the purpose of catching the flooding wave and not to propagate beyond the flooded area. Figure 11 illustrates the storm control mechanism and initiation of flood control. The set of actions are the same for both the data frames and the inhibitor frames.

\subsection{Variables and justifications}

Following variables may be set with default values when the endcast scheme is installed into devices by assuming or by letting the user select a MANET scenario, which is described by the approximate number of devices and the physical area.

\subsubsection{Chalone wait}

Chalone wait can be recognized as a random assessment delay (RAD) defined in [9]. Common practice of setting a RAD is to specify a maximum delay $\left(T_{\max }\right)$ for a node to receive rebroadcast of neighbors and RAD is chosen randomly from the uniform distribution of delays within 0 to $T_{\max }$ [9]. This randomization avoids collisions. However, chalone wait should be large enough to receive as many neighbor responses as possible and should be small enough to give an acceptable end to end delay for the frame.

$T_{\max }$ is set to $0.001,0.01,0.05$ and $0.1 \mathrm{~s}$ in the simulation study to compare broadcast protocols in [9]. $T_{\max }$ is set to $0.01 \mathrm{~s}$ in the simulation setup in [24]. Delay and backoff intervals in a Rene mote testbed for flooding experiments are randomly picked from the range 0.006 to $0.01 \mathrm{~s}$. In [25] the maximal transmission latency is calculated by dividing the transmission range from transmission speed. IEEE 802.11 standard specifies the speed of radio waves propagating in free space as $300 \mathrm{~m} / \mu \mathrm{s}$. We set the $T_{\max }$ by dividing the maximum distance between a node and it's neighbors by the lowest transmission speed. This results in the worst case time duration for an immediate neighbor of a node to communicate with it.

\subsubsection{Chalone threshold}

Chalone threshold should be an optimal value so as not to cause too many redundant rebroadcasts and for the data frame to reach the destination. This threshold depends on the node density, and an adaptive counterbased flooding mechanism is proposed in [26] where the threshold is a function of the size of neighborhood (n). According to [26], the lowest possible threshold is 2 and the highest possible is $n+1$. When $n$ is small the threshold should be larger and vice versa. The threshold should be set to an initial value and then it should automatically adapt to the frame count according to a suitable function $C(n)$. It should also have an upper limit, and according to [26], only a few rebroadcasts can be saved by setting the threshold to be greater than 6 in sparse networks. An abstract shape for $C(n)$ is proposed, and the exact shape is derived using an experiment in [26].

\subsubsection{Inhibitor wait}

Inhibitor wait should be less than the chalone wait to ensure that the inhibitor has the necessary speed to catch the flooding wave that propagates beyond the destination. This is similar to the recall protocol proposed by Gao et al. [27]. It is also advisable to predict and initiate the inhibition process earlier rather than to wait until the destination is reached.

\subsubsection{Data buffer}

According to the protocol shown in Fig. 11, data frames should be buffered until a decision is made whether to rebroadcast or discard it. Time duration for buffering the frame and how the buffer should be maintained depends on a number of factors. For example, a frame should be kept in the buffer at least for chalone wait time added with some processing overhead time.

\subsubsection{Frame log}

A log record of a frame should reside in a log maintained by a node for a maximum period of time equal to the lifetime of the frame. Lifetime of a frame in the case of end to end data delivery is only until it is delivered to the destination. Therefore, the longest lifetime of a log record is the time taken by a frame to travel from one end to the other end of a network with all the delays included. Hence the lifetime of a log record depends both on the diameter of the network in terms of the number of hops and waiting times at each hop.

The cost of maintaining a frame log is comparable to the cost of performing simple flooding. In simple flooding, a node has to record the source address and the sequence number in a log for checking whether subsequently received frames are duplicates. In the proposed endcast scheme a log record includes the destination 
address of the message and few more state bits in addition to the sequence numbers logged in simple flooding. Details of the fields of a log record shown in Fig. 12 are as follows:

Frame ID is the combination of source MAC, destination $\mathrm{MAC}$, and the sequence number. An end to end data frame is uniquely identified by a Frame ID, and its corresponding inhibitor also carries the same Frame ID.

Data state carries one of the states from the set: unknown, originated, buffered, rebroadcast, discarded, and accepted. The state unknown is when the node has never received a particular data frame so that there is no record of that particular frame in the log. The state originated refers to the case that the frame is originated at the node in concern. An incoming data frame from the physical layer will be buffered when it is received for the first time and this state is identified as buffered. A buffered data frame will be discarded when more duplicates than the threshold are received subsequently. When redundant frames are received while the frame is in data buffer, the neighbor count in the log record will be updated before discarding the frame. When the frame is no longer in the buffer so that it is finished with processing, the redundant frames will be silently discarded. The discarding of a data frame results in discarded state. If the data was rebroadcast then the log record carries rebroadcast state. The data state in the log record becomes accepted if the particular data frame is destined to the node and received for the first time.

Inhibitor state carries one of the states from the set: unknown, originated, buffered, rebroadcast, discarded, and the explainations are similar to data states except that the states are relevant to inhibitor messages. Similar to the data frames, the inhibitors incoming from the physical layer are either buffered or discarded. While an inhibitor is already in the buffer subsequently received inhibitors with same data frame ID result in an increment in the inhibitor count before getting discarded. If an inhibitor is completely processed according to the log and no longer resides in the buffer, redundant inhibitors are silently discarded.

The important parameters of the proposed endcast scheme are summarized in Table 5.

\section{Theoretically modeling the proposed endcast scheme}

The probabilities of node-to-node transmission and multihop frame propagation are derived using the previously introduced process described in the Section 4. The proposed endcast scheme uses counter-based flooding for storm control. The edges of flooding graph are indexed by time of reception of frames to analyze the effect of the
Table 5 Summary of parameters in proposed Endcast scheme

\begin{tabular}{ll}
\hline Parameter & Description \\
\hline Chalone wait & $\begin{array}{l}\text { Time for a data message to wait in } \\
\text { buffer for neighbor information }\end{array}$ \\
Chalone threshold & $\begin{array}{l}\text { Threshold for the number of copies } \\
\text { of the same message (data or } \\
\text { inhibitor) to receive for taking the } \\
\text { rebroadcast decision } \\
\text { Inhibitor wait }\end{array}$ \\
Message ID & $\begin{array}{l}\text { Timfor in buffer for neighbor } \\
\text { information }\end{array}$ \\
Message log & $\begin{array}{l}\text { Source address, destination address } \\
\text { and sequence number }\end{array}$ \\
& $\begin{array}{l}\text { Fields: timestamp, message ID, } \\
\text { data state, inhibitor state, neighbor } \\
\text { count, inhibitor count }\end{array}$ \\
Data and inhibitor buffers & $\begin{array}{l}\text { Temporary store of messages until a } \\
\text { rebroadcast decision is made }\end{array}$ \\
\hline
\end{tabular}

assessment delay $T_{R A D}$ as previously explained in Modeling efficent flooding schemes. Depending on the assignement of values to $T_{R A D} \mathrm{~S}$, the structure of the flooding graph changes in this proposed endcast scheme.

\subsection{Modeling counter-based storm control mechanism}

Chalone concentration $C$ is a discrete random variable, which can be described by a probability mass function $f(c)$. Hence, $P=P\{C<K\}$ is the cumulative distribution function $F(C)$ for the chalone threshold $K$. By the definition:

$$
F(C)=\sum_{i=0}^{K} f(c)
$$

In order to construct the probability mass function $f(c)$, it is required to find the probabilities that a node receive $c$ number of copies of the same frame where $0 \leq c<N$ and $N$ is the number of tentative rebroadcasting nodes. The probability that a node will record a particular counter value at a given time depends on the relative positions of the tentative rebroadcasters at that time. Consider the original broadcast of a frame by node A in Fig. 13. The neighbors of A, namely, B, C, and D will receive this frame. Let all of them rebroadcast the frame and then any node in the dark gray area will record a counter value of three as it receives from $\mathrm{B}, \mathrm{C}$, and $\mathrm{D}$ after receiving originally from A. Similarly, the white area in A's range receives only from A and records a counter value of zero, light gray area records a counter value of one and gray area records a counter value of two.

$$
P\{C=c\}=\frac{A_{c}}{A_{\text {reg }}}
$$

Probability $P\{C=c\}$ that a node records a particular counter value $c$ is the set of points in the region 


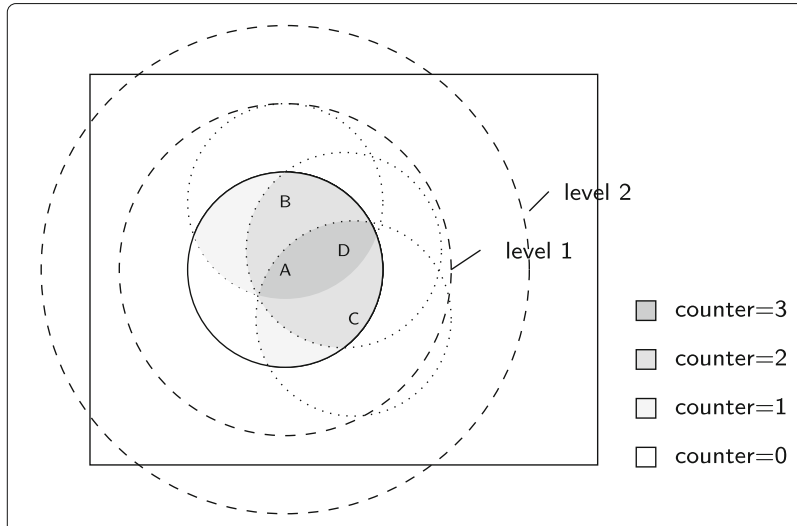

Fig. 13 Geometric interpretation of counter-based flooding that will be covered by $c$ number of circles divided by all possible points in the region. This implies that the probability should be expressed in terms of areas as in Eq. 34 where $A_{c}$ denotes the intersection area of $c$ circles and $A_{\text {reg }}$ is the total area of the region in which the circles are deployed. The centers of the circles are the potential rebroadcasters.

Calculating the area of intersection for more than three circles is considered practically unsolvable as a closed form expression because there are an infinite number of possible configurations for how the circles can intersect. Librino et al. [28] proposed an algorithm that iteratively computes the intersection area of an arbitrary number of circles in terms of matrix manipulations. This algorithm can be used to derive the $\operatorname{PMF} f(c)$ for a specified location map of dense population of nodes in a MANET since the manipulation of large matrices is becoming practical with advances in high performance computing.

\subsection{Modeling the effect of mobility on counter-based mechanism}

The configuration of the radio range circles vary with the mobility of nodes as illustrated in the sample network in Figs. 14 and 15. Change of PMF $f(c)$ due to node mobility is used to analyze the effect of mobility on proposed endcast scheme. The Librino algorithm is run on the snapshots of the network to calculate $f(c)$ repetitively and thereby estimate a mobility dependent probability $P_{t x}$ of rebroadcasting.

6.3 Modeling the effect of chalone wait and inhibitor wait In order to analyze the protocol in time domain the graph model should be modified by indexing the edges with timestamp as shown in Fig. 16. Accordingly, every time a message is rebroadcast a new time index is added to the graph either on an existing edge or by inserting a new edge. Therefore, the total number of messages accumulated in the network is given

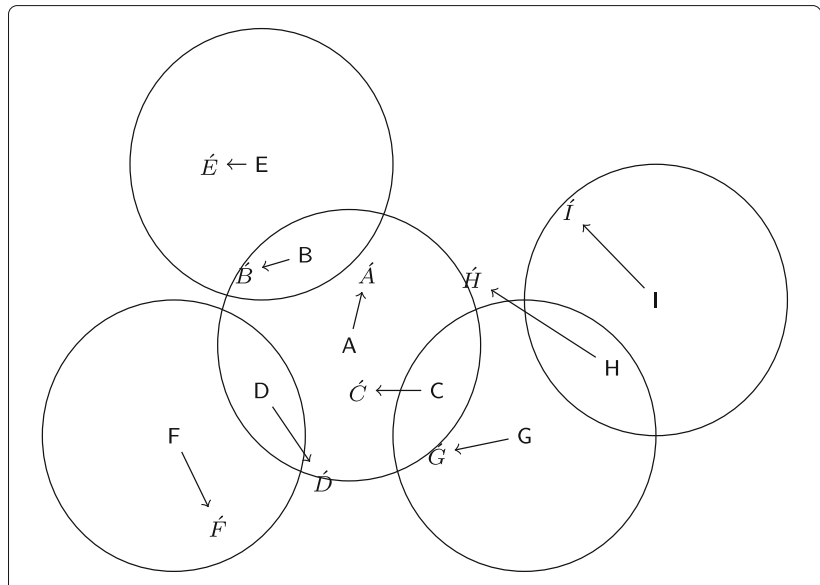

Fig. 14 Problem of endcast in the presence of mobility

by the total number of time indices. The number of indices plotted against time allows for storm and flood analysis.

Consider the 20-node sample network given in Fig. 17. Assume node h1 is the source and h17 is the destination. The original broadcast of the source will be received by all its neighbors inside the solid circle at time $t_{0}$. It will take the chalone wait time $\left(t_{c w}\right)$ for any one of these nodes to rebroadcast or discard the message. However, h17 as the destination will immediately broadcast the inhibitor at time $t_{i 0}$. The neighbors of h17 will receive this inhibitor (within the dotted circle). The nodes in the intersection between the solid and dotted circles are those who have received the data from $h 1$ (source) and the relevant inhibitor from h17 (destination). These nodes will wait for the inhibitor wait time $\left(t_{i w}\right)$ and rebroadcast the inhibitor. Node h6 does not rebroadcast the inhibitor because the relevant data is unknown for h6. According to Table 6, node h1 has the smallest $t_{i w}$ and therefore h1 will be the first to rebroadcast the inhibitor. Since $t_{i w}<<$ $t_{c w}$, the nodes in the solid circle receive the inhibitor before $t_{c w}$ expires for the level 1 rebroadcasting and thus all refrain from rebroadcasting. This way the inhibitor wave propagates only towards the area of the network that is already flooded with data and faster than the propagation of data.

Figure 18 shows the total number of data messages and inhibitor messages in the network against time assuming chalone wait is around $0.5 \mathrm{~s}$ and the inhibitor wait is around $0.1 \mathrm{~s}$. Fast propagation of inhibitor succeeds in limiting the number of data messages to 10 that resulted from initial broadcast in this case. The number of inhibitors is 17 but the inhibitor is a small size message that consists only of a header to carry the ID of the data message being inhibited and own type as inihibitor. 


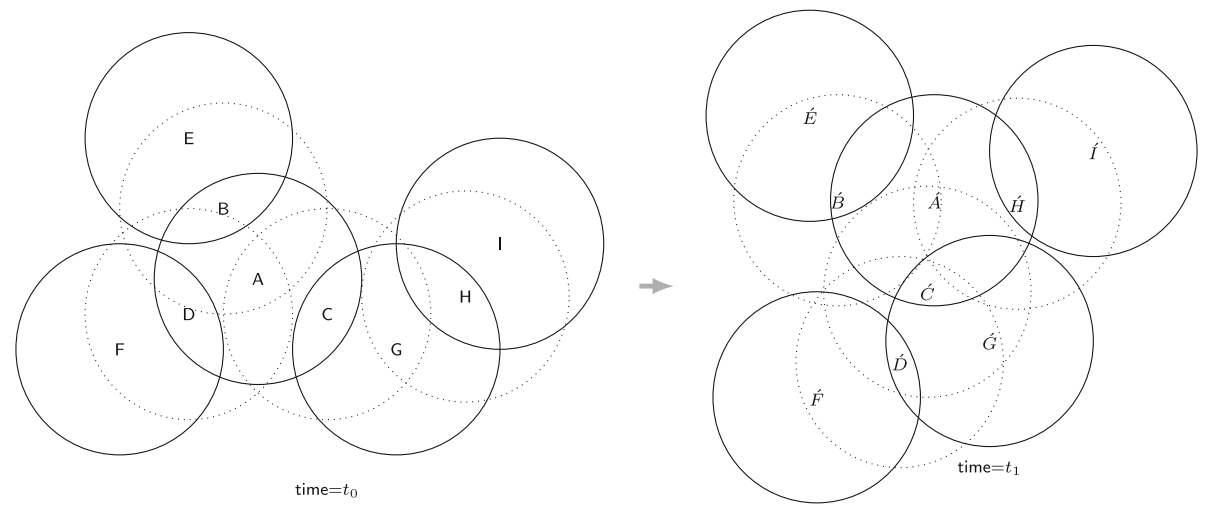

Fig. 15 Network configurations at two time instances while the nodes are moving

\section{Theoretical results and discussion}

$$
N_{T}=P_{S} N+P_{S} N \sum_{i=1}^{l-1}\left(\beta P\{C<K\} P_{b} N\right)^{i}
$$

Reachability of the proposed endcast scheme was calculated according to Eq. 35 using the parameter set in Table 7. In Eq. 35, we do not consider variable coverage areas by different rebroadcast levels and thus we use $\beta$ instead of $\beta_{i}$ for simplifying the analysis.

The resulting plot of reachability against different retransmission levels is shown in Fig. 19. According to Fig. 19, a low chalone threshold such as one or two results in reachability that asymptotically reach a relatively small percentage of around $20 \%$ of the nodes. A threshold value of 3 and above is capable of delivering the messages to all the nodes in the network. When the threshold is increased from three, reachability curve becomes steeper so that the nodes are reached by few rebroadcast levels. This result can be used to estimate an optimal threshold value for a target MANET application scenario.

\section{Simulation study of the proposed endcast scheme}

We refer to our earlier proposed endcast scheme as CA protocol to denote its cellular automata-based design. This section presents the simulation study of the CA protocol. The experiments were conducted on OMNET++ (version 4.4.1) using INET (version 2.1) and MiXiM (version 2.3) frameworks [29].

The simulations were designed for evaluating the performance of CA protocol in comparison with Sequence Number Controlled Flooding (SNCF) or simple flooding. We selected or defined following metrics after a broad survey of available performance metrics:
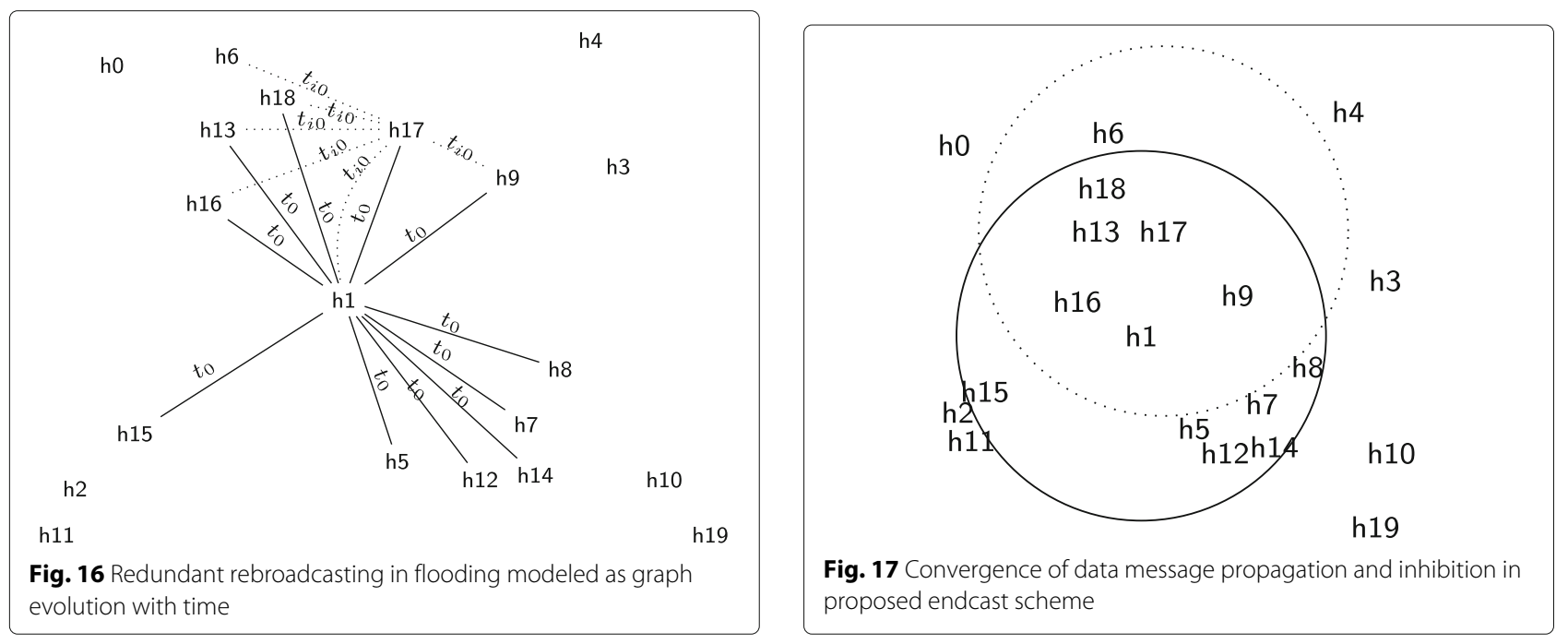
Table 6 Order of expiring chalone wait and inhibitor wait (e.g., h0 has the smallest wait and h20 has the largest wait)

\begin{tabular}{lllllllllllllllllllll}
\hline Node & h0 & h1 & h2 & h3 & h4 & h5 & h6 & h7 & h8 & h9 & h10 & h11 & h12 & h13 & h14 & h15 & h16 & h17 & h18 & h19 \\
\hline RAD order & 1 & 2 & 3 & 4 & 5 & 6 & 7 & 8 & 9 & 10 & 11 & 12 & 13 & 14 & 15 & 16 & 17 & 18 & 19 & 20 \\
\hline
\end{tabular}

Redundancy overhead $\left(\mathrm{ROH}_{u f}\right)$ : We refer to redundant copies of the same data frame accumulated in the network as redundancy overhead for endcast by modifying the metric $\mathrm{ROH}$ used by Reina et. al [30].

Saved ReBroadcasts (SRB): SRB is defined by Tseng et. al [26] as the ratio between the number of nodes that refrain from rebroadcasting and the number of nodes that receive a frame. Accordingly, SRB also measures the redundant rebroadcasts but in terms of number of nodes. $S R B=\frac{r-t}{r}$, where $r$ is the number of receiving nodes and $t$ is the number of transmitting nodes [26].

REachability ( $R E$ and $\left.R E_{u f}\right)$ : Efficient flooding schemes impose a risk of not reaching the destination in the process of suppressing the rebroadcasts. Therefore, it is essential to evaluate flooding schemes in terms of reachability. Reachability is defined for network wide broadcasts as the ratio between the number nodes reached $(r)$ by the flooding scheme and the number of reachable nodes $(e)$ by topology. Thus, $R E=\frac{r}{e}$. However, we achieve endcast using efficient flooding by purposely curtailing the redundant rebroadcasts. The aim of the CA protocol is to reach the destination as opposed to reaching all nodes in the network in traditional use of flooding schemes. Therefore, we defined Reachability for endcast as the ratio between the number

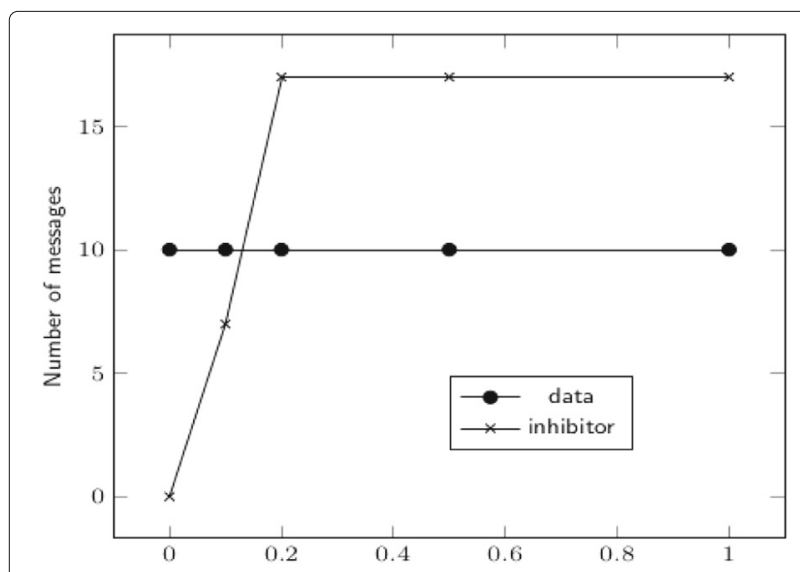

Fig. 18 Total number of data and inhibitor messages in the network with time of reached node pairs $\left(r_{u f}\right)$ by the flooding scheme and the number of all reachable pairs $e_{u f}$. Thus $R E_{u f}=\frac{r_{u f}}{e_{u f}}$. We use both of these definitions in our simulation study.

Flooding completion time (FCT): Flooding schemes involve a random assessment delay (RAD) [9]. For example a node that runs simple flooding waits a random period of time to avoid collisions. CA protocol waits for both to avoid collisions and to receive redundant frames to extract neighbor information. These waiting periods directly impact the end to end delay for a frame to travel from source to destination. Therefore, latency is an important metric to compare performance of flooding schemes especially when used for end to end data delivery. Flooding completion time (FCT) is the interval between the time at which the flooding is initiated and the time at which the final rebroadcast occurs or the final decision is made not to rebroadcast.

\subsection{Experimental design}

We conducted two sets of experiments: (i) based on ideal wireless conditions and (ii) to capture realistic network conditions. Ideal wireless conditions imply that there are no collisions, no interferences and even no propagation delays. Nodes are also assumed to be static. The protocols are tested in such a scenario in order to isolate protocol specific issues such as impact of the protocol logic on performance, for example, reachability. Further, in ideal wireless condition the frames are instantaneously delivered to all the neighbors. It results in maximum

Table 7 Parameter set for the reachability analysis of an example MANET

\begin{tabular}{ll}
\hline Parameter & Value \\
\hline Playground & $100 \mathrm{~m} \times 100 \mathrm{~m}$ \\
Total nodes & 300 \\
Transmission radius & $40 \mathrm{~m}$ \\
Average neighborhood & 100 \\
Listening probability, $p$ & 0.0005 \\
Slots per frame Tx time, $\tau$ & 100 \\
Rebroadcasting probabilities $P_{t x}$ & $0.04,0.06,0.08$ and 0.11 \\
Mobility & No \\
\hline
\end{tabular}




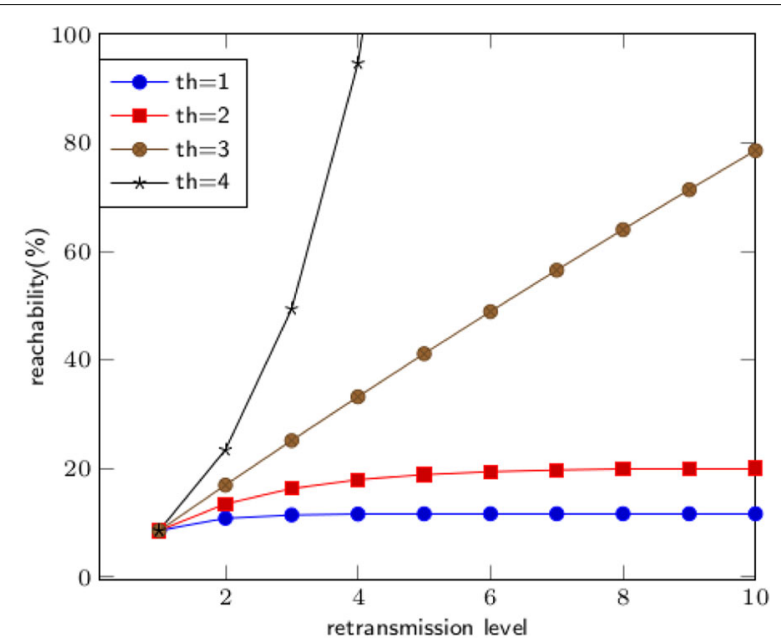

Fig. 19 Reachability with retransmission level in a MANET having density 100 frame counts whereas in realistic wireless conditions some frames may not be delivered, for example, due to collisions. Therefore, ideal wireless scenario results in the maximum reception of frames as well as maximum number of rebroadcasts so that it will give the worst performance in terms of redundant frames for any flooding scheme. As a first step of evaluating the protocols, we collected only the number of frames with varying network parameters such as number of nodes and neighborhood size.

Realistic wireless network conditions include collisions, interferences, and propagation delays of wireless links. In multihop networks, there are additional problems such as hidden terminal problem and exposed source problem. Furthermore, CA protocol when targeted at application scenarios, such as in a disaster situation, the protocol must be tested in the presence of node mobility. Hence, this set of experiments was designed to assess protocol performance in the presence of node mobility, realistic wireless conditions, and varying network densities.

\subsubsection{Experiments based on ideal wireless conditions}

Blind rebroadcasting causes a node to rebroadcast every time a message is received. This shows the closest analogy to uncontrolled biological cell proliferation and we based the CA protocol on blind rebroadcasting. However, in general, SNCF is the implemented version of blind rebroadcasting with an improvement such that the frame is rebroadcast only once by any node. Therefore, we compare CA protocol with both blind rebroadcasting and SNCF in these experiments.

These experiments correspond to the simulation parameters given in Table 8 . We randomly place nodes on the same playground of size $600 m \times 400 m$ with each node having transmission radius of $200 \mathrm{~m}$. This playground size and transmission range are comparable to number of similar simulation studies $[5,31,32]$ and within the ranges in Table 9. Chalone timeout is set at a small value of $0.1 \mu \mathrm{s}$ since the frames are delivered instantaneously in ideal wireless conditions.

\subsubsection{Experiments based on realistic wireless conditions}

The physical layer settings of commodity wireless devices conform to IEEE 802.11 wireless standards and we selected IEEE 802.11g implementation of MIXIM-INET for the following experiments. IEEE 802.11's medium access control (MAC) is called Distributed Coordinate Function (DCF) which is a CSMA/CA scheme according to [33]. We consider only the basic DCF in standard IEEE 802.11 MAC because broadcasts are handled using basic DCF and flooding schemes are broadcast based [34].

\section{Simulation results and discussion}

Redundancy, reachability and latency aspects of the CA protocol against SNCF were studied by configuring the simulation with ideal wireless conditions in Table 8, realistic wireless conditions in Table 10 and with network topologies described in Tables 11, 12, and 13.

\subsection{Simulation results}

\subsubsection{Redundancy overhead}

Experiment 1 We placed 20 to 1000 nodes on a playground of size $600 m \times 400 m$ with each node having a $200-\mathrm{m}$ transmission range in ideal wireless conditions. We counted the total number of frames $\left(\mathrm{ROH}_{u f}\right)$ received by all the nodes in the network by the time the destination is reached, for the same source destination pair when the simulation was run for 10 different node placements under the ideal wireless conditions. We averaged the results over these 10 simulation runs and a plot of the results with 99\% confidence interval is shown in Fig. 20. Note that the CA protocol was configured without an inhibition scheme to stop the propagation of flooding wave after the destination is reached and also the threshold is set to be one.

Table 8 Simulation parameters for experiments in ideal wireless conditions

\begin{tabular}{ll}
\hline Parameter & Value \\
\hline Playground size & $600 \mathrm{~m} \times 400 \mathrm{~m}$ \\
Transmitter range & $200 \mathrm{~m}$ \\
Chalone timeout & $0.1 \mu \mathrm{s}$ \\
Chalone threshold & 1 to 3
\end{tabular}


Table 9 Surveyed simulation parameters

\begin{tabular}{lll}
\hline Parameter & Values & \\
\hline Playground size & Minimum & $250 \mathrm{~m} \times 250 \mathrm{~m}$ \\
& Maximum & $5500 \mathrm{~m} \times 5500 \mathrm{~m}$ \\
Number of nodes & 200 to 1000 & \\
$\begin{array}{l}\text { Network density } \\
\text { (neighbors per node) }\end{array}$ & 4 to 20 \\
Radio range $(\mathrm{m})$ & 88 to 500 & \\
Data rate (Mbps) & 1 and 2 \\
Packet size (bytes) & $64,100,256,280,1024$ \\
\hline
\end{tabular}

According to Fig. 20, the CA protocol outperforms both blind rebroadcasting and SNCF protocol for all numbers of nodes shown. For example, in a MANET with 100 nodes, blind rebroadcasting caused the network to receive the same frame around 2440 times on average, while SNCF and CA protocol caused 1870 and 1000 frames respectively. Thus the CA protocol saves nearly $60 \%$ of redundant rebroadcasts compared to blind rebroadcasting whereas the saving is nearly $45 \%$ compared to SNCF. Also for 1000-node network the frame count was more than 280,000 for blind rebroadcasting, around 210,000 for SNCF and only around 70,000 frames for CA protocol. CA protocol in small MANETs of 20 nodes also shows a saving of redundant rebroadcasts around $40 \%$ and $10 \%$ compared to blind rebroadcasting and SNCF protocol respectively.

Blind rebroadcasts are unacceptable for dense MANETs in terms of redundant rebroadcasts as the growth of the curve is steeply exponential. Though SNCF is slightly better compared to blind rebroadcasting, it also shows an exponential growth. CA on the other hand shows a slow growing curve thus making it a better protocol for all network sizes shown. Hence the CA protocol reduces storm situation better than SNCF.

Experiment 2 This experiment analyzed the redundancy overhead of CA protocol compared to SNCF in the presence of realistic wireless conditions in Table 10 with

Table 10 IEEE 802.11 parameters [146]

\begin{tabular}{lll}
\hline Parameter & Value & \\
\cline { 2 - 3 } & $2.4 \mathrm{GHz}$ band & $5.0 \mathrm{GHz}$ band \\
\hline Slot time & Short $=9 \mu \mathrm{s}$, long $=20 \mu \mathrm{s}$ & $9 \mu \mathrm{s}$ \\
SIFS & $10 \mu \mathrm{s}$ & $16 \mu \mathrm{s}$ \\
DIFS & Short $=28 \mu \mathrm{s}$, long $=50 \mu \mathrm{s}$ & $34 \mu \mathrm{s}$ \\
Bitrate & $2 \mathrm{Mbps}$ & $2 \mathrm{Mbps}$ \\
CWmin & 15 & 15 \\
CWmax & 1023 & 1023 \\
Retry limit & 7 (short) & \\
& 4 (long) & \\
\hline
\end{tabular}

Table 11 Network densities of simulated topologies

\begin{tabular}{lll}
\hline Playgrond size & Average node degree & Median node degree \\
\hline $20 \mathrm{~m} \times 20 \mathrm{~m}$ & 299.0 & 299 \\
$50 \mathrm{~m} \times 50 \mathrm{~m}$ & 256.5 & 260 \\
$100 \mathrm{~m} \times 100 \mathrm{~m}$ & 105.0 & 104 \\
$150 \mathrm{~m} \times 150 \mathrm{~m}$ & 52.8 & 53 \\
$200 \mathrm{~m} \times 200 \mathrm{~m}$ & 31.7 & 31 \\
$250 \mathrm{~m} \times 250 \mathrm{~m}$ & 21.0 & 20 \\
$300 \mathrm{~m} \times 300 \mathrm{~m}$ & 15.1 & 14 \\
$350 \mathrm{~m} \times 350 \mathrm{~m}$ & 11.4 & 11 \\
$400 \mathrm{~m} \times 400 \mathrm{~m}$ & 8.9 & 9 \\
\hline
\end{tabular}

network densities in Table 11. We placed 300 nodes in playgrounds of sizes ranging from $20 \mathrm{~m} \times 20 \mathrm{~m}$ to $400 m \times 400 m$ so that the network density in terms of average neighbors per node varies from 10 to 300 .

Figure 21 shows variation of the redundancy overhead in terms of total number of frames accumulate in the network $\left(R O H_{u f}\right)$ for CA and SNCF protocols until the end of all the protocol events. Results are interpreted in two ways: redundant frames with the playground size and the same with node densities.

According to Fig. 18, the total number of accumulated redundant frames at the end of the simulation is low at about 1000 frames for CA protocol for all the network densities and playground sizes and it is a significantly better performance compared to SNCF, which records over 2000 to 50,000 frames for the same network configurations. The growth of redundant frames for SNCF is unacceptably high as it grows from around 2500 frames to 50,000 when the network density increases from 10 to 300 neighbors per node. However, CA protocol shows almost constant number of redundant frames for all node densities shown. This is because the destination is reached by the time around 1000 redundant frames are recorded in the network in all network configurations in CA protocol and thereafter, the inhibition scheme of the CA protocol effectively stops the propagation of frames throughout the network.

Experiment 3 We used the same test configuration in experiment 2 and obtained the number of nodes that

Table 12 Topologies with constant network density

\begin{tabular}{lll}
\hline Playground & Nodes & Density \\
\hline $20 \mathrm{~m} \times 20 \mathrm{~m}$ & 11 & 10.00 \\
$85 \mathrm{~m} \times 85 \mathrm{~m}$ & 20 & 9.61 \\
$150 \mathrm{~m} \times 150 \mathrm{~m}$ & 50 & 10.70 \\
$220 \mathrm{~m} \times 220 \mathrm{~m}$ & 100 & 10.55 \\
\hline
\end{tabular}


Table 13 Simulation parameters for experiments in realistic wireless conditions

\begin{tabular}{lll}
\hline Parameter & Value & \\
\hline Number of nodes & 300 & \\
Transmission range & $40 \mathrm{~m}$ & Disaster site \\
Playground sizes & Meeting room & $450 \mathrm{~m} \times 450 \mathrm{~m}$ \\
Chalone wait & $20 \mathrm{~m} \times 20 \mathrm{~m}$ & \\
Flooding wait & 0 to $6 \mathrm{~s}$ & \\
Threshold & 0 to $6 \mathrm{~s}$ & \\
\hline
\end{tabular}

received the frame and the number of nodes that rebroadcast the frame in the networks. We calculated $S R B$ as a percentage. The plot of SRB against both the playground size and the network density is shown in Fig. 22.

It was observed that in $\mathrm{SNCF}$, all nodes receiving a frame did rebroadcast it thus giving no saving of rebroadcasts. CA protocol performs better for all node densities and all playground sizes with over $60 \%$ saving recorded for all these network configurations. Interestingly, SRB increases when the network density increases in CA protocol. The saving in rebroadcasts sharply increases by about $30 \%$ when the node density increases from 10 to 50 .

\subsubsection{Reachability}

The performance gain in terms of redundant rebroadcasts is due to suppression of rebroadcasts using the threshold driven counter-based mechanism. However, suppression of rebroadcasts imposes a risk of not reaching the destination. Therefore, reachability was analyzed according to the original definition, $R E=\frac{r}{e}$ where $r$ is the number of reached nodes and $e$ is the number of reachable nodes. We find $r$ using simulations whereas $e$ is found using graph theoretic approach as follows:

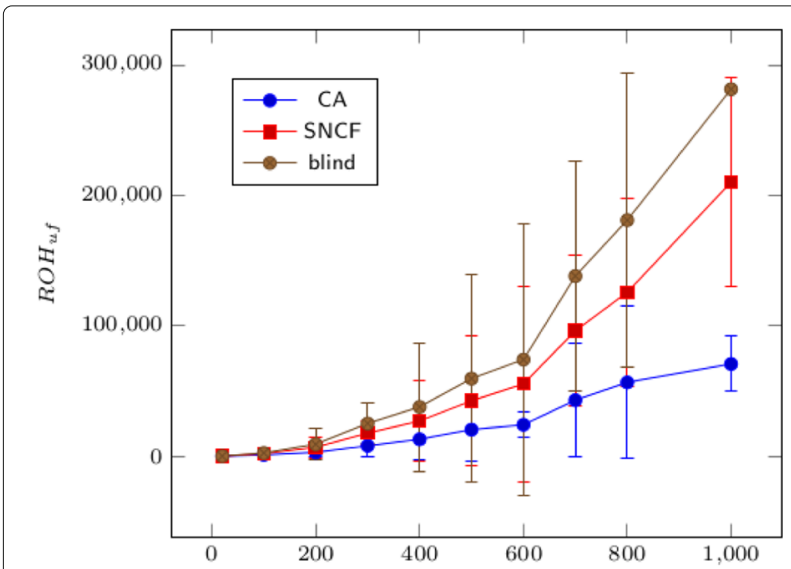

Fig. 20 Total number of redundant frames received in the network $\left(R O H_{u f}\right)$ until the destination is reached for blind, SNCF, and CA protocols in ideal wireless conditions
We model the MANET as a graph in which vertices are the mobile nodes and the edges denote the availability of a direct wireless link between nodes at a given point in time. We first take the Euclidean distance between every node pair and then determine the immediate neighborhood by considering the transmission ranges. It is analogous to super imposing the communication ranges of nodes on the physical location map of the network. The resulting graph can be identified as a transmission graph. In order to evaluate the reachability of this graph, we use Floyd-Warshall algorithm that provides simplicity, parallel computation for all node pairs and low pre-processing needs. If a path can be found by the algorithm then we can conclude that a node can reach any other node by simple flooding.

Experiment 4 We configured the simulation as in experiment 2 and obtained number of reachable nodes using Floyd-Warshall all pair shortest path algorithm. Then we executed CA and SNCF protocols to obtain the number of reached nodes. After that we calculated $R E$ and plotted the results against both the network densities and playground sizes. Figure 23 shows reachability as a percentage, achieved by CA protocol when the playground size and the node density varies. SNCF gave $100 \%$ reachability for all node densities and playground sizes considered and this data is not shown in the graph for the clarity of the data points. According to Fig. 23 the reachability of CA protocol decreases when the node density decreases or the playground size increases and this is for the smallest possible threshold value which is 1 .

However, this reduction in RE should not be interpreted as a weakness of the CA protocol because it purposely curtails the rebroadcasts and is aimed at delivering end to end data. Therefore, we run experiment 5 to measure $R E_{u f}$ to analyze the performance of CA protocol in end to end data delivery.

Experiment 5 In this experiment we maintained the average network density at around 10 neighbors per node by varying the number of nodes from 11 to 100 and varying the playground size from $20 m \times 20 m$ to $220 m \times 220 m$ as shown in Table 12. The experiment was on realistic wireless conditions from Table 10. When all node pairs are considered, 100-node network requires to be simulated with end to end data delivery for $9900(100 \times 100-$ 100) node pairs. These tests required heavy computational costs, therefore we had to semi automate and repeat the tests manually in batches. Therefore, only 3 data points were obtained as shown in Fig. 24.

It was observed according to Fig. 24a that the reachability was $100 \%$ for smaller networks of size 10 nodes for SNCF and CA protocol even with threshold of one. We get this complete reachability with CA when threshold is 

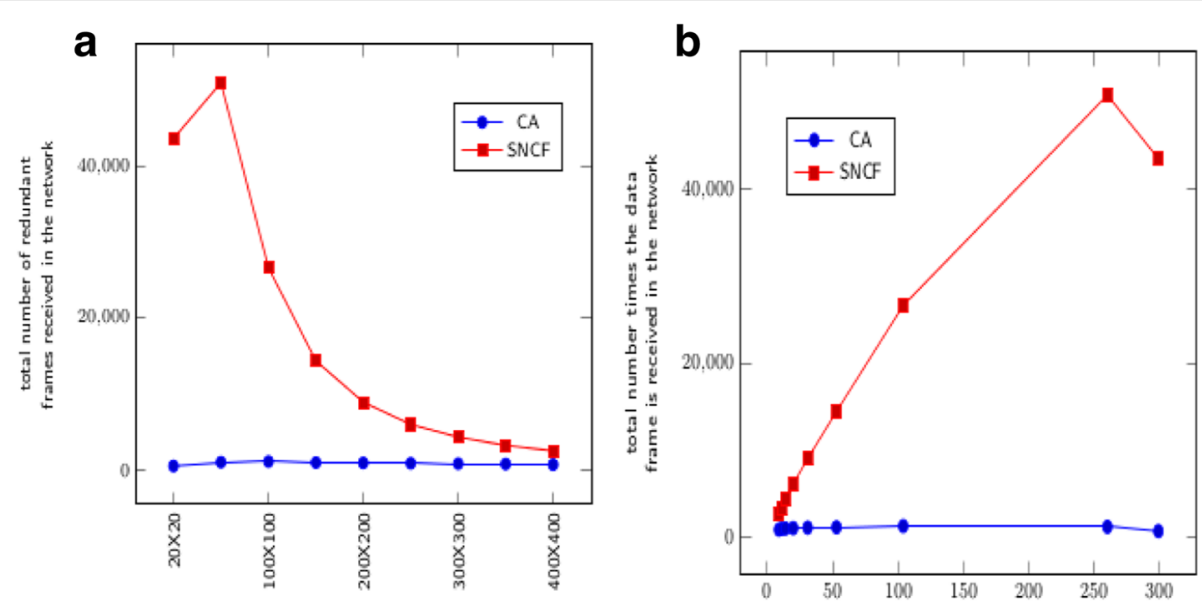

Fig. 21 Redundant frames until the end of events in SNCF and CA protocols. a Against playground size. b Against node density

one, while saving about $80 \%$ of redundant frames compared to SNCF as in Fig. 24b. However, when the network is large and nodes are dispersed in a larger area, the frame should propagate in a multihop manner to reach some node pairs. Depending on which nodes decide to rebroadcast in CA protocol, the destination may not be reached in some cases. This is visible in the 50-node network where reachability of $\mathrm{CA}$ is only about $75 \%$ when the threshold is one whereas SNCF shows around $90 \%$ reachability.

According to Fig. 24a reachability increases when the threshold increases in realistic network conditions also. For example, reachability improves from 75 to $90 \%$ in the 50 -node network by increasing threshold from one to two. This significant reachability improvement is gained while still saving around $80 \%$ of redundancy overhead compared to SNCF.
The redundancy-reachability tradeoff can be analyzed using the ratio between RE and $R O H_{u f}\left(\mathrm{RE} / R O H_{u f}\right)$. For example, for the static 50-node network scenario in Fig. 24 this ratio is 0.34 for SNCF, 0.97 for CA protocol with threshold 1 and 0.88 for CA protocol with threshold 2 . Accordingly when threshold increases the ratio decreases and ultimately CA protocol acts as SNCF when threshold is increased beyond the maximum neighborhood in the network.

\subsubsection{Latency}

Experiment 6 We configured the simulation as in experiment 2, where CA and SNCF protocols are simulated on realistic network conditions with 300 nodes placed on playgrounds of sizes $20 m \times 20 m$ to $400 m \times 400 m$. We obtained flooding completion time (FCT) by taking
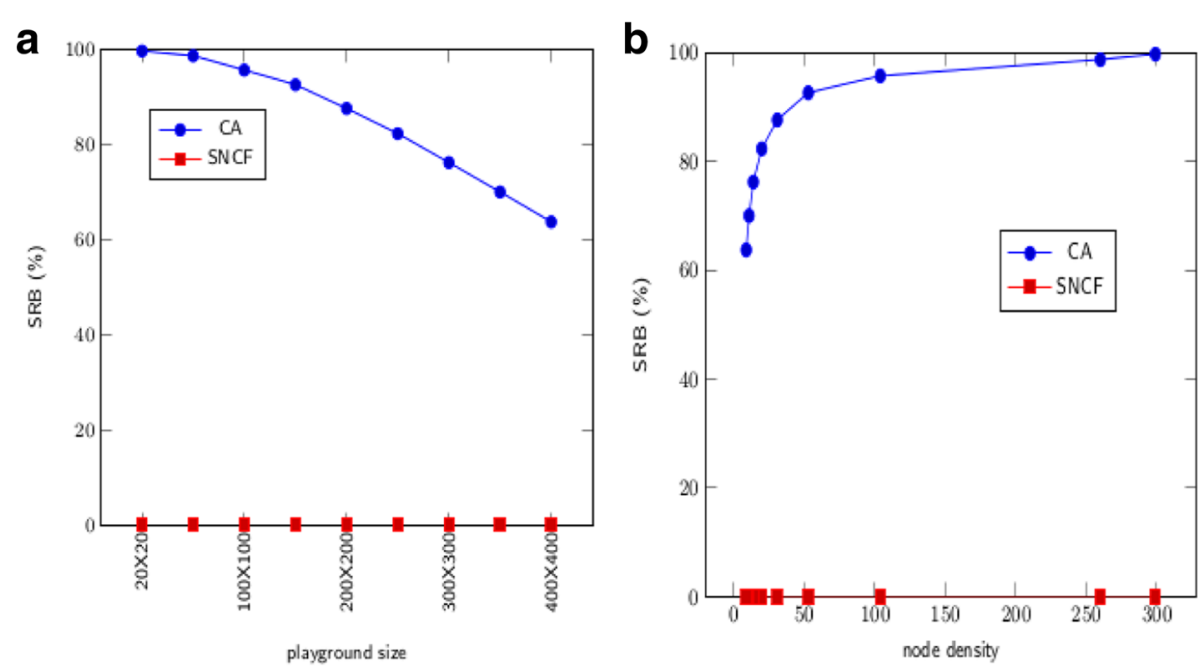

Fig. 22 Saved rebroadcasts (SRB) for CA and SNCF protocols for a 300-node network. a Against playground size. b Against node density 

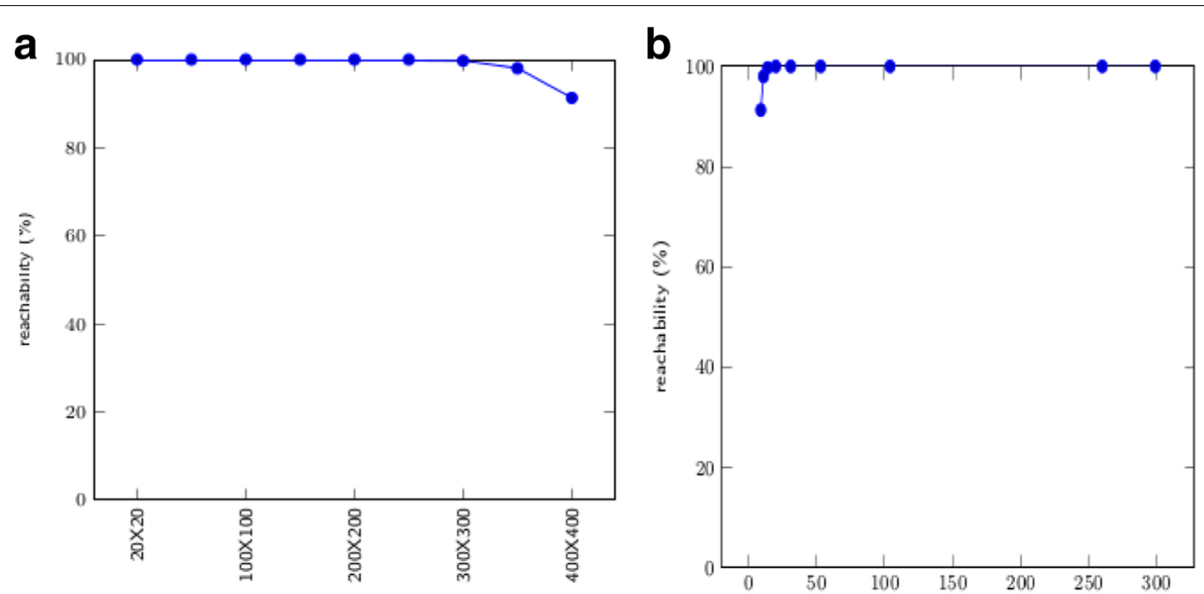

Fig. 23 Reachability of SNCF and CA protocols. Reachability is 100\% for SNCF for all the considered network configurations. a Against playground size. b Against node density

the difference between the timestamp at which the frame gets originated and the timestamp at the end of the simulation. Accordingly, FCT consists of chalone wait periods in CA protocol and random wait periods in SNCF, backoff periods and propagation delays of IEEE 802.11 MAC and physical layer. The ACK timeouts do not apply in IEEE 802.11 MAC specifications as broadcasts are not acknowledged. Only single stage backoff at the transmitting node applies in detecting an idle channel. However, the MAC level delays are at microsecond scale whereas RAD times such as chalone wait in CA protocol and random delay in SNCF are much higher at $0.6 \mathrm{~s}$. Therefore, the latency metrics are governed by RAD time.

The plot of FCT resulting from CA protocol is comparable with that resulting from SNCF according to Fig. 25 as the two curves are almost superimposed on one another. However, there is slightly higher FCT recorded by SNCF compared to CA protocol for highly dense networks according to Fig. 25b. For example, in networks of around densities of 250 and 300 neighbors per node, SNCF takes about 5 more seconds to finish its frame propagation than CA protocol. On the other hand for low density networks such as 10 neighbors per node, the latency is higher in CA protocol with a gap of about $25 \mathrm{~s}$.

\subsection{Discussion}

\subsubsection{Effect of storm control}

CA protocol controls the redundant rebroadcasting of frames using a counter-based mechanism and this was termed storm control. According to simulation results, CA protocol saves over $45 \%$ of redundant frames compared to SNCF even when it runs without inhibition
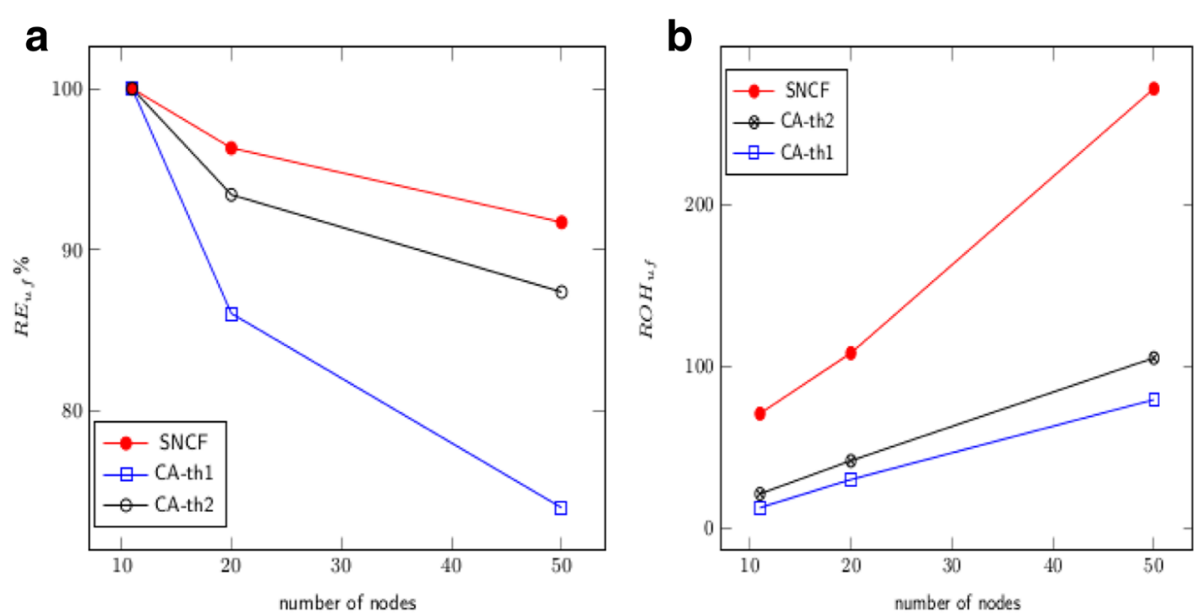

Fig. 24 Reachability $\left(R E_{u f}\right)$ and redundancy overhead $\left(R O H_{u f}\right)$ of endcast with SNCF and CA protocols when the network size varies with network density kept constant at 10. a Reachability. b Redundancy overhead 

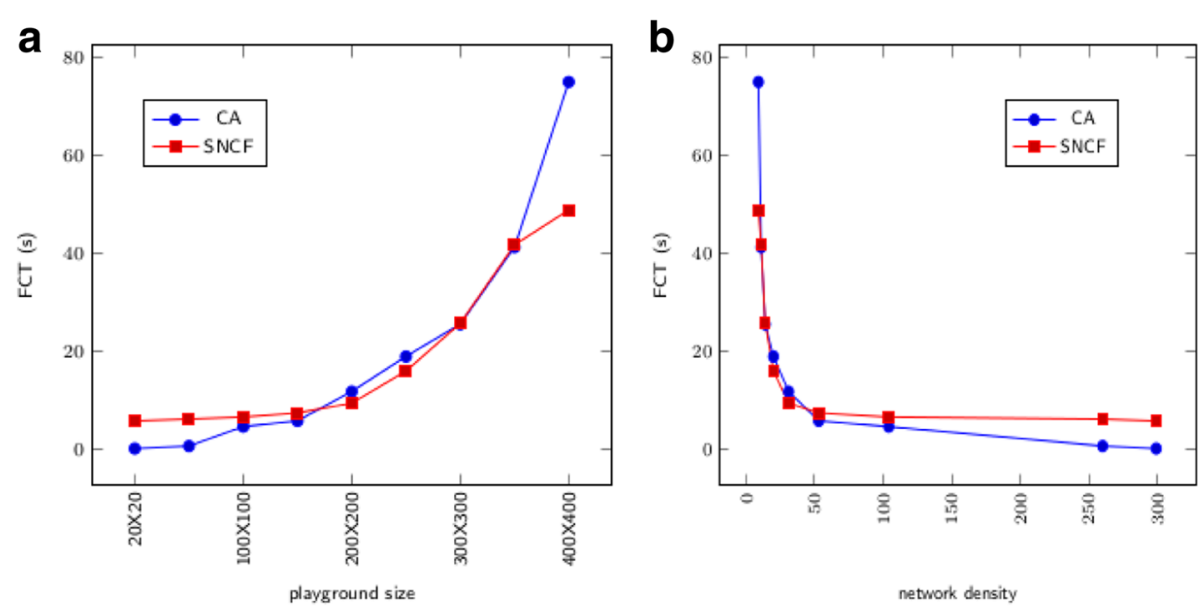

Fig. 25 Latency of CA protocol in terms of flooding completion time (FCT) in comparison with simple flooding. a Against playground size. b Against node density

scheme for flood controlling (Fig. 20). This saving is recorded when the threshold for storm control was one and the number of nodes were over 100 . The nodes were placed on a playground of size $400 m \times 600 m$ with each node having $200 m$ transmission range. Further, when the number of nodes were increased to 1000 , the saving of redundant frames increases to $65 \%$.

With the inhibition scheme in operation the saving in redundant frames is over $50 \%$ (Fig. 18) for networks of densities 10 through 300 of average number of neighbors per node. These network densities were obtained by placing 300 nodes in playgrounds of sizes $20 m \times 20 m$ to $400 m \times 400 m$ and configuring each node to have power-saving radio range of $40 \mathrm{~m}$. It was observed that the number of frames remain almost constant at around 1000 throughout the total range of network densities in CA protocol whereas the number increased from 2000 to 50000 in SNCF. This implies the saving in redundant frames in CA protocol increases from $50 \%$ at network density of 10 to about $98 \%$ at network density of 300 with respect to the redundant frames caused by SNCF. These statistics for variety of network configurations can be interpreted as evidence for the effectiveness in saving redundant frames in the network by the storm control using counterbased flooding and the flood control using negative acknowledgement.

\subsubsection{Effect of inhibition scheme}

The effectiveness of inhibition scheme is further evaluated by comparing the total number of redundant rebroadcasts with and without inhibition until all the protocol events are over. Figure 20 shows variation of number of redundant frames with varying number of nodes, when the CA protocol operates with and without inhibition scheme in ideal wireless conditions with simulation parameters configured according to Table 8.

Figure 26 shows almost constant growth rate of about eight frames per node in the plot for no inhibition. This growth is due to the continuous propagation of the frame throughout the network after reaching the destination. When the inhibition scheme is in place there is almost no growth in the number of frames when the number of nodes increases as the frame stops propagating soon after the destination is reached. The destination is reached in all networks considered within the total frame count of 100. For example in a 200-node network, the given destination was reached by the time the total number of frames was counted as 84 when the inhibition scheme was in operation. The frame count was 1405 when the inhibition was not used. This is around 15 times the recorded number of frames when the inhibition scheme is used.

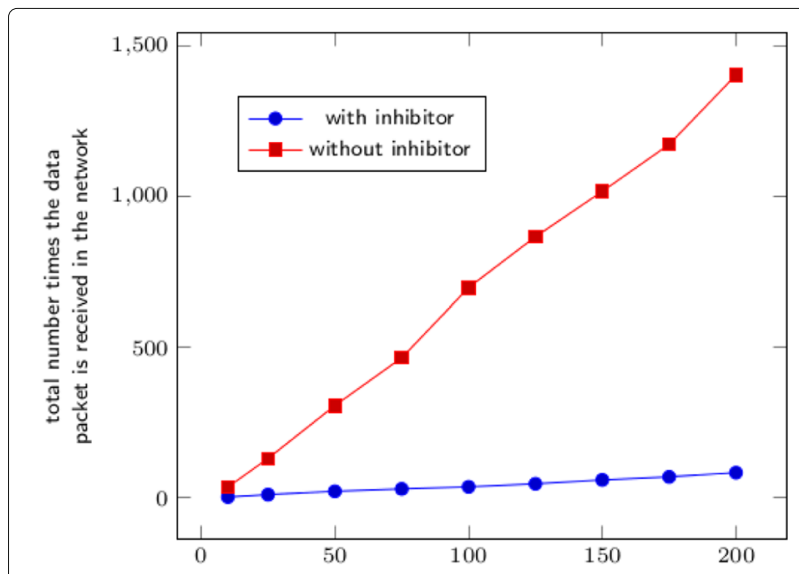

Fig. 26 Number of redundant rebroadcasts until the end of all events in CA protocol with and without inihibition scheme 
This shows that the inhibition scheme significantly saves redundant frames accumulated after reaching the destination. Therefore, inhibition scheme satisfactorily controls flood situation.

\subsubsection{Effect of mobility}

It is essential to evaluate MANET protocols in the presence of mobility. Random Way Point (RWP) mobility is extensively applied in wireless network simulations [35-38]. In RWP mobility model, a node selects a random starting position and a random point in the area as the next waypoint. Then it moves towards the selected waypoint at a randomly picked speed between a minimum and a maximum speed. After reaching the determined waypoint it waits for a time duration, normally distributed between a minimum and a maximum, and repeats the process with next selected waypoint [36]. If the target application scenario is for human mobility as in the case of managing a disaster relief camp, minimum and maximum speeds are selected as 1 to $2 \mathrm{~m} / \mathrm{s}$ and the pause time is between 0 and $0.5 \mathrm{~s}$. These values are in line with similar studies that capture human mobility scenarios [35-38].

Figure 27 corresponds to a network density of 10 as defined in terms of average neighborhood per node. The simulation was run by increasing the number of nodes and the playground size while maintaining this network density. Transmission radius was also kept fixed at power saving range of $40 \mathrm{~m}$. Therefore, the network diameter increases in terms of hop count when the number of nodes increases.

The simulations were run by configuring the network to be static as well as by allowing the nodes to move according to RWP mobility with speed from 1 to $2 \mathrm{~m} / \mathrm{s}$ and pause time from 0 to $0.5 \mathrm{~s}$. We tested SNCF protocol and CA protocol with both static and mobile network configurations for varying network sizes. CA protocol was tested for threshold values of one and two, which are denoted as th 1 and th 2 in Fig. 27.

Figure 27a shows the number of redundant frames accumulated in the network until the destination is reached when the network size is increased while keeping the average network density constant at 10. Accordingly, SNCF shows the highest frame counts and it grows faster than for CA protocol. For example, in a 50-node network SNCF caused accumulation of around 250 frames while CA protocol resulted in a number less than half of that. Furthermore, frame count increases from around 75 to 250 by SNCF in static network when the network size increases from around 10 to 50 nodes. The growth of frame count is even steeper for SNCF when the nodes are mobile as it increases from about 75 to 400. In contrast CA protocol shows only an increase from about 20 to 150 when the network size increases from 10 to 50 nodes.

In the presence of mobility, there is a higher frame count than in static networks for all protocol settings. However, the redundant frames caused by SNCF with mobility shows a considerable increase compared to the case of SNCF in static networks, for example, in a 50-node network, the mobility of nodes caused about $60 \%$ increase in redundant frames. In contrast, mobility of nodes does not cause considerable increase in redundant frames compared to static networks when CA protocol is in operation.

Figure 27b shows how the reachability varies with network size for static nodes and mobile nodes in SNCF, CA protocol with threshold 1 and CA protocol with threshold 2 . Here, the reachability is according to the definition of RE, redefined for end to end data delivery by considering source-destination pairs.

As per Fig. 27b, mobility seems to assist frame propagation rather than hinder it because the reachability of SNCF
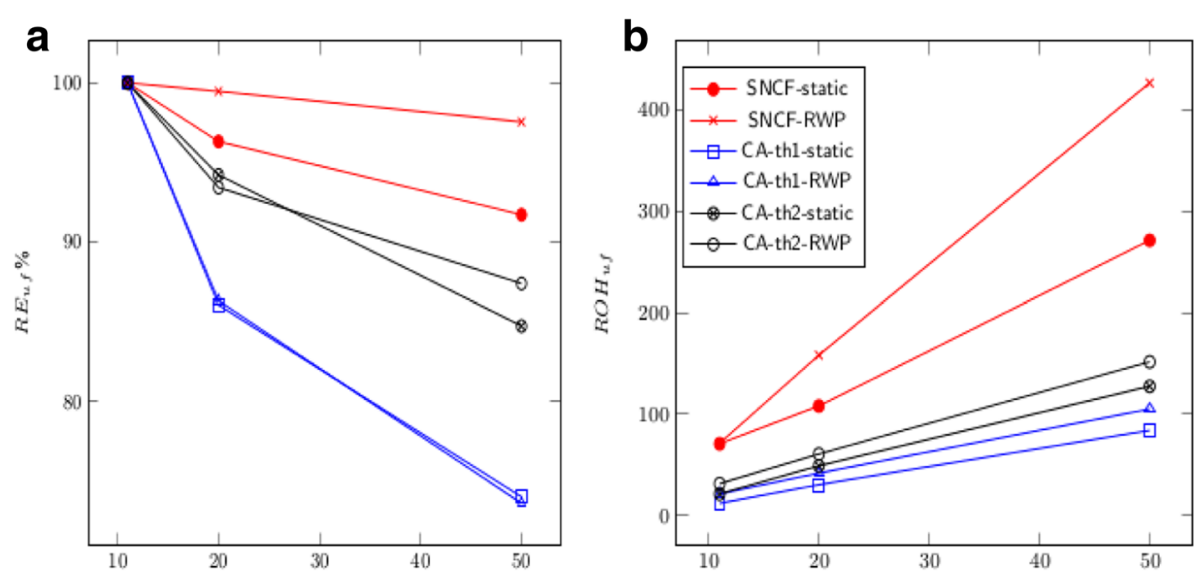

Fig. 27 Redundancy overhead and reachability of endcast until the destination is reached for SNCF and CA protocols with mobility when the threshold of CA protocol is set at 1 and 2 (th 1 and th2). a Reachability. b Redundancy 
is significantly higher in mobile network compared to static network. Reachability is almost constant at around $100 \%$ for all the network sizes with mobile nodes for SNCF except for a slight decrease in higher network sizes. In contrast, in static networks reachability of SNCF drops from 100 to around $95 \%$ when network size increases from 10 to 50 . A general perception is that mobility of nodes are disruptive in data communication in wireless networks and makes it challenging to device routing mechanisms. That may be true for routing schemes that set up virtual backbones and assign key nodes to act as routers. However, the flooding schemes considered here, can be considered to leverage mobility to enhance data delivery. This is in line with the argument supporting the branch of MANET study known as opportunistic networks in which mobility is considered an opportunity for delivering data using store and carry behavior of nodes. CA protocol with threshold of two, also shows a slightly higher reachability in mobile networks compared to static networks at least for higher network sizes. CA protocol with threshold of one does not show any difference in reachability when operating on static and mobile networks.

\subsection{Effect of chalone threshold}

In experiment 5, we evaluated $R E_{u f}$ and $R O H_{u f}$ in networks of different sizes but with constant network density of around 10 neighbors per node. When the two metrics are compared side by side as in Fig. 24, by slightly increasing the threshold, reachability could be improved considerably. For example, in a 50-node network the reachability could be increased by about $15 \%$ when the threshold was increased simply from one to two. Moreover, this slight increment in threshold does not increase the redundancy overhead in a considerable amount, for example, in the same 50-node network the number of frames increased only by about $6 \%$ when the threshold increased from one to two.

Accordingly, as chalone threshold becomes higher, for the CA protocol, the reachability-redundancy tradeoff becomes smaller and CA protocol acts similar to SNCF. Furthermore, mobility assists communication in flooding but at the expense of redundancy.

\section{Conclusions}

A key objective of this research was to build a theoretical basis to analyze the problem of routing in MANETs approached from a new paradigm that we term as mobilestateless paradigm, which is completely different from traditional MANET routing. In this model, nodes do not keep any state information and they can become stateless. If they are allowed to blindly rebroadcast every received data frame then it results in unrestricted node mobility. However, blind rebroadcasting is unacceptably inefficient. Instead, we can use simple flooding, in which a node rebroadcasts a frame only when it is received for the first time. There are flooding schemes proposed in the literature that allow only a sub set of the nodes to rebroadcast and these schemes are referred to as efficient flooding. Conventional use of any kind of network flooding is to disseminate a message to all the nodes in a network. In contrast, we propose to use flooding for end to end data delivery. We did not come across any other work that attempts end to end data delivey using flooding with a theoretical analysis.

We leveraged efficient flooding as an end to end data delivery mechanism conforming to mobile-stateless routing paradigm and we term it as endcast. We also developed a model to analyze endcast schemes in terms of evolution of a graph $G(V, E)$, where the set of vertices $V$ represent the nodes and an edge $(u, v) \in E$ is added when a frame is received by a node $v$ due to rebroadcasting of another node $u$ during the propagation of a frame via flooding. In our model, the number of edges are estimated using geometric analysis of the network.

Our model captures the major problems in flooding, namely, the broadcast storm problem and the situation that we call broadcast flood problem that is particularly observed in endcast. Broadcast storm problem refers to the situation where excessive number of redundant frames are transmitted all over the network causing heavy contention and collisions. This situation is already researched in the literature aiming at improving conventional use of flooding for network wide broadcast. When flooding is used for end to end data delivery, it is wasteful to leave the frames to propagate beyond the destination and we refer to this situation as broadcast flood problem.

The theoretical model that we built for analyzing endcast is capable of:

- Modeling the propagation of data via flooding schemes including simple flooding, probabilistic flooding, and counter-based flooding

- Analyzing flooding in networks with static nodes as well as mobile nodes

- Estimating the level of broadcast storm problem and broadcast flood problem

- Modeling the physical and MAC layer impacts such as hidden terminal problem

Our model extends the work of Viswanath-Obraczka [2] for analyzing multihop flooding in static networks as follows:

- We calculate the additional area covered by each level of rebroadcast geometrically so that $\beta$ varies from level to level.

- We incorporate time domain in the graph to model flooding schemes that involve an assessment time before making a rebroadcast decision. 
- We model node mobility using the time domain representation of the graph as well as the mobility-dependent variation of coverage areas.

- Instead of using a random $P_{t x}$ we interpret $P_{t x}$ in terms of different parameters in flooding schemes, for example we derive a $P_{t x}$ for counter-based flooding based on the counter value and the threshold.

\section{Additional file}

Additional file 1: These are .dat files used for plotting graphs using pgfplot package. The commands for plotting are commented inside figure commands as the online editorial manager could not compile the commands. The data file for Fig. 10 (edgeGrow.dat); the data file for Fig. 19

(ReachabilityVsLevel.dat); a data file for Fig. 20 (CA.dat); a data file for Fig. 20 (SNCF.dat); a data file for Fig. 20 (blind.dat); a data file for Fig. 18 (CAUntilEnd.dat); a data file for Fig. 18 (sncfUntilEnd.dat); a data file for Fig. 18 (CApkts.dat); a data file for Fig. 18 (SNCFpkts.dat); a data file for Fig. 22 (CaSRB.dat); a data file for Fig. 22 (SncfSRB.dat); a data file for Fig. 22 (CaSRBnei.dat); a data file for Fig. 22 (SncfSRBnei.dat); a data file for Fig. 23 (ReachTopo.dat); a data file for Fig. 23 (CAReach.dat); a data file for Figs. 24 and 27 (eval.dat); a data file for Fig. 24 (eval-redframes.dat); a data file for Fig. 25 (DelayCAnSNCF.dat); a data file for Fig. 25 (DelayCAnSNCFwithDen.dat); the data file for Fig. 26 (withNwithoutlnhib.dat); the data file for Fig. 18 (timeConverge.dat). (ZIP 6 kb)

\section{Funding}

This research was conducted for the fulfillment of PhD in Computer Science and Engineering from the University of Moratuwa. The Department of Computer Science and Engineering provided necessary facilities and guidance for the research work.

\section{Authors' contributions}

The first author SJS conducted the research and prepared the manuscript under the supervision of the second author CDG. Both authors read and approved the final manuscript.

\section{Competing interests}

The authors declare that they have no competing interests.

\section{Publisher's Note}

Springer Nature remains neutral with regard to jurisdictional claims in published maps and institutional affiliations.

\section{Received: 20 December 2016 Accepted: 16 August 2017} Published online: 31 August 2017

\section{References}

1. M Abolhasan, T Wysocki, E Dutkiewicz, A review of routing protocols for mobile ad hoc networks. Ad Hoc Netw. 2(1), 1-22 (2004)

2. KViswanath, K Obraczka, Modeling the performance of flooding in wireless multi-hop ad hoc networks. Comput. Commun. 29(8), 949-956 (2006)

3. LWu, PK Varshney, Performance analysis of csma and btma protocols in multihop networks (i) - single channel case. Inf. Sci. 120(1), 159-177 (1999)

4. YC Tseng, SY Ni, YS Chen, JP Sheu, The broadcast storm problem in a mobile ad hoc network. Wirel. Netw. 8(2-3), 153-167 (2002)

5. C Ho, K Obraczka, G Tsudik, K Viswanath, in The 3rd ACM International Workshop on Discrete Algorithms and Methods for Mobile Computing and Communications. Flooding for reliable multicast in multi-hop ad hoc networks (ACM, 1999), pp. 64-71

6. A Rahman, W Olesinski, P Gburzynski, in IEEE International Workshop on in Wireless Ad-Hoc Networks. Controlled flooding in wireless ad-hoc networks (IEEE, 2004), pp. 73-78
7. H Takagi, L Kleinrock, Optimal transmission ranges for randomly distributed packet radio terminals. IEEE Trans. Commun. 32(3), 246-257 (1984)

8. R Rajaraman, Topology control and routing in ad hoc networks: a survey. ACM SIGACT News. 33(2), 60-73 (2002)

9. B Williams, T Camp, in 3rd ACM International Symposium on Mobile Ad HoC Networking and Computing. Comparison of broadcasting techniques for mobile ad hoc networks (ACM, 2002), pp. 194-205

10. SCho, JP Hayes, in IEEE Wireless Communications and Networking Conference. Impact of mobility on connection in ad hoc networks, vol. 3 (IEEE, 2005), pp. 1650-1656

11. JC Lui, J Baron, Mechanisms limiting body growth in mammals. Endocr. Rev. 32(3), 422-440 (2011)

12. GP Finkielstain, P Forcinito, JC Lui, KM Barnes, R Marino, S Makaroun, $\checkmark$ Nguyen, JE Lazarus, O Nilsson, J Baron, An extensive genetic program occurring during postnatal growth in multiple tissues. Endocrinology. 150(4), 1791-1800 (2009)

13. PJ Bryant, P Simpson, Intrinsic and extrinsic control of growth in developing organs. Q. Rev. Biol. 59(4), 387-415 (1984)

14. WS Bullough, Mitotic and functional homeostasis:a speculative review. Cancer Res. 25(10), 1683-1727 (1965)

15. H Onda, A new hypothesis on mitotic control mechanism in eukaryotic cells: cell-specific mitosis-inhibiting protein excretion hypothesis. Theor. Biol. 77(3), 367-377 (1979)

16. SJ Lee, Regulation of muscle mass by myostatin. Annu. Rev. Cell Dev. Biol. 20, 61-86 (2004)

17. K Elgjo, KL Reichelt, Chalones: from aqueous extracts to oligopeptides. Cell Cycle. 3(9), 1208-1211 (2004)

18. D Bakthavatsalam, DA Brock, NN Nikravan, KD Houston, RD Hatton, RH Gomer, Chalones: from aqueous extracts to oligopeptides. Cell Sci. $121,2473-2480$ (2008)

19. AR Kansal, S Torquato, GR Harsh, EA Chiocca, TS Deisboeck, Simulated brain tumor growth dynamics using a three-dimensional cellular automaton. J. Theor. Biol. 203(4), 367-382 (2000)

20. Y Lee, S Kouvroukoglou, LV McIntire, K Zygourakis, A cellular automaton model for the proliferation of migrating contact-inhibited cells. Biophys. J. 69, 1284-1298 (1995)

21. T Alarcóna, HM Byrneb, PK Mainia, A cellular automaton model for tumour growth in inhomogeneous environment. J. Theor. Biol. 225(2), 257-274 (2003)

22. O Mireira, A Deutsch, Cellular automaton models of tumor development: A critical review. Advs. Complex Syst. 5(2/3), 247-268 (2002)

23. NH Packard, S Wolfram, Two-dimensional cellular automata. J. Stat. Phys. 38(5/6), 901-946 (1985)

24. A Mohammed, M Ould-Khaoua, L Mackenzie, An efficient counter-based broadcast scheme for mobile ad hoc networks. Formal Methods and Stochastic Models for Performance Evaluation. (Springer, 2007), pp. 275-283

25. S Pleisch, M Balakrishnan, K Birman, RV Renesse, in The 7th ACM International Symposium on Mobile Ad Hoc Networking and Computing. MISTRAL: efficient flooding in mobile ad-hoc networks (ACM, 2006), pp. 1-12

26. YC Tseng, SY Ni, EY Shih, Adaptive approaches to relieving broadcast storms in a wireless multihop mobile ad hoc network. IEEE Trans. Comput. 52(5), 545-557 (2003)

27. J Gao, L Guibas, N Milosavljevic, J Hershberger, in The 6th ACM International Conference on Information Processing in Sensor Networks. Sparse data aggregation in sensor networks (ACM, 2007), pp. 430-439

28. F Librino, M Levorato, M Zorzi, An algorithmic solution for computing circle intersection areas and its applications to wireless communications. Wirel. Commun. Mob. Comput. 14(18), 1672-1690 (2014)

29. OpenSim Ltd., OMNet++ (2001). https://omnetpp.org/. Accessed 19 Sept 2016

30. DG Reina, SL Toral, P Johnson, F Barrero, A survey on probabilistic broadcast schemes for wireless ad hoc networks. Ad Hoc Netw. 25, 263-292 (2015)

31. Y Sasson, D Cavin, A Schiper, Probabilistic broadcast for flooding in wireless mobile ad hoc networks. IEEE Wirel. Commun. Netw. 2, 1124-1130 (2003)

32. Y Yi, M Gerla, TJ Kwon, in IEEE International Conference on Communications (ICC 2003). Efficient flooding in ad hoc networks: a comparative performance study, vol. 2 (IEEE, 2003), pp. 1059-1063

33. G Bianchi, leee 802.11-saturation throughput analysis. IEEE Commun. Lett. 2(12), 318-320 (1998) 
34. IEEE 802.11 Working Group and others, IEEE Standard for Information Technology-Telecommunications and information exchange between systems-Local and metropolitan area networks-Specific

requirements-Part 11: Wireless LAN Medium Access Control (MAC) and Physical Layer (PHY) specifications Amendment 6: Wireless Access in Vehicular Environments. IEEE Std. 802(11) (2010)

35. M Cornils, M Bahr, T Gamer, in European Wireless Conference (EW). Simulative analysis of the hybrid wireless mesh protocol (HWMP) (IEEE, 2010), pp. 536-543

36. M Ikeda, T Honda, $T$ Oda, S Sakamoto, L Barolli, in IEEE 28th International Conference on Advanced Information Networking and Applications. Analysis of WMN-GA simulation results: WMN performance considering stationary and mobile scenarios (IEEE, 2014), pp. 337-342

37. N Sabah, A Hocanin, Gamma random waypoint mobility model for wireless ad hoc networks. Int. J. Commun. Syst. 26(11), 1433-1445 (2013)

38. O Younes, N Thomas, Analysis of the expected number of hops in mobile ad hoc networks with random waypoint mobility. Electron. Notes Theor. Comput. Sci. 275, 143-158 (2011)

\section{Submit your manuscript to a SpringerOpen ${ }^{\circ}$ journal and benefit from:}

- Convenient online submission

- Rigorous peer review

- Open access: articles freely available online

- High visibility within the field

- Retaining the copyright to your article

Submit your next manuscript at $\boldsymbol{s p r i n g e r o p e n . c o m ~}$ 